\title{
Underdetermined-Order Recursive Least-Squares Adaptive Filtering: The Concept and Algorithms
}

\author{
Buyurman Baykal, Member, IEEE, and Anthony G. Constantinides, Senior Member, IEEE
}

\begin{abstract}
The concept of underdetermined recursive leastsquares (URLS) adaptive filtering is introduced. In particular, the URLS algorithm is derived and shown to be a direct consequence of the principle of minimal disturbance. By exploiting the Hankel structure of the filter input matrix, the fast transversal filter (FTF) version of the URLS algorithm (URLS-FTF) is derived including sliding window and growing window types, which allow alteration of the order of the URLS algorithm (which is equivalent to the linear prediction order of the input) in real time. The computational complexity is reduced to $O(N)+O(m)$, where $N$ is the adaptive filter length, and $m$ is the order of the URLS algorithm. In addition, the efficient URLS (EURLS) algorithm, which does not compute the filter coefficients explicitly, thereby significantly reducing the computational load, is presented. Some earlier adaptive algorithms such as the averaged LMS, filtered-X LMS, and fast conjugate gradient are shown to be suboptimal approximations of the URLS algorithm. Instrumental variable approximations are also discussed. The URLS algorithm has a whitening effect on the input signal, which provides immunity to the eigenvalue spread of the input signal correlation matrix. Although the algorithm is sensitive to observation noise, it has good tracking characteristics, and tradeoffs can be found by tuning the step size. The utility of the URLS algorithms, in its basic form and FTF realization, depends heavily on the practical applicability of the $m$ th-order sliding window estimate of the covariance matrix and $m$ th-order FTF relations. The feasibility of the URLS family in practical applications is demonstrated in the simulations, which include channel equalization and acoustic echo cancellation in hands-free terminals with real data sets.
\end{abstract}

\section{INTRODUCTION}

$\mathbf{L}$ EAST-SQUARES parameter estimation techniques have a long and successful history since Gauss' first formulation. The basic idea is to choose the parameter estimate that minimizes an error-squared sum criterion that is related to a set of overdetermined linear equations, the coefficients of which are unknown parameters. In this paper, we look at the problem of least-squares parameter estimation from a different perspective. We assume that the error criterion is related to an underdetermined set of linear functions of the unknown parameters and wish to obtain a recursive estimator while maintaining the underdetermined nature of the problem as it evolves because of some desirable properties, such as good tracking, that may accrue. Such a formulation appears to have received little exposure. The earliest work in 1984 is by Ozeki in [1] and with some applications in [2]. Modifications and incremental improvements have also appeared in [3]

Manuscript received March 25, 1995; revised July 22, 1996. The associate editors coordinating the review of this paper and approving it for publication were Dr. and Mrs. Stelio Mangiola.

The authors are with the Department of Electrical and Electronic Engineering, Imperial College, London SW7 2BT, England.

Publisher Item Identifier S 1053-587X(97)01186-0. and [4], while Slock [5] has derived independently some of the essential components of this initial work. Montazeri and Duhamel have published some results very recently [6], [7]. In [7], they used a block-based approach that enabled the use of Levinson recursions without increasing the computational load. In their other paper [6], they proposed a modification for the calculation of the inverse of the covariance matrix to overcome ill conditioning that may arise in practical applications, which we also discuss in Section VI. The same paper also includes an exponentially weighted approximate version of the EURLS algorithm presented in Section IV. Their approach is based on $m \ll N$ and, hence, is dependent on the relative sizes of prediction order and adaptive filter size. The URLS-type algorithms have gained practical importance recently, particularly in acoustic echo cancellation [6], [8], [9], which have reported encouraging results. In this paper, we also demonstrate the feasibility of the URLS algorithms in practical applications.

The following issues are specifically addressed in this paper:

- The concept of underdetermined recursive least-squares filtering is introduced from first principles to fill the gap between normalized least mean square (NLMS) and recursive least squares (RLS) algorithms and defined formally, which has been lacking up to now.

- Fast URLS algorithms are derived.

- Various approximations are considered, and some earlier adaptive filtering algorithms are shown to belong to a class that consists of approximations of the URLS algorithm.

- The URLS algorithm is assessed by simulations under various conditions.

Fig. 1 presents a conceptual comparison of the various adaptive algorithms and their optimal properties within a leastsquares sense. The step-size $\mu$ is assumed to be in the stable range. The URLS algorithm with $\mu=1$ lies in the optimal region when the equations solved to obtain the estimate are fewer than the number of unknown parameters. The fast conjugate gradient algorithm [10] is a suboptimal approximation to it. The suboptimal approximation of the RLS algorithms (the fast quasi Newton (FQN) algorithm [11]) updates the covariance matrix of the input in every $N$ instants by assuming that the covariance matrix changes slowly with time.

A newer concept in adaptive filtering, namely, the subspace tracking, offers an alternative solution in the case where there are dominant eigenvalues of the covariance matrix [12]-[15]. Subspace tracking is usually a computationally expensive 


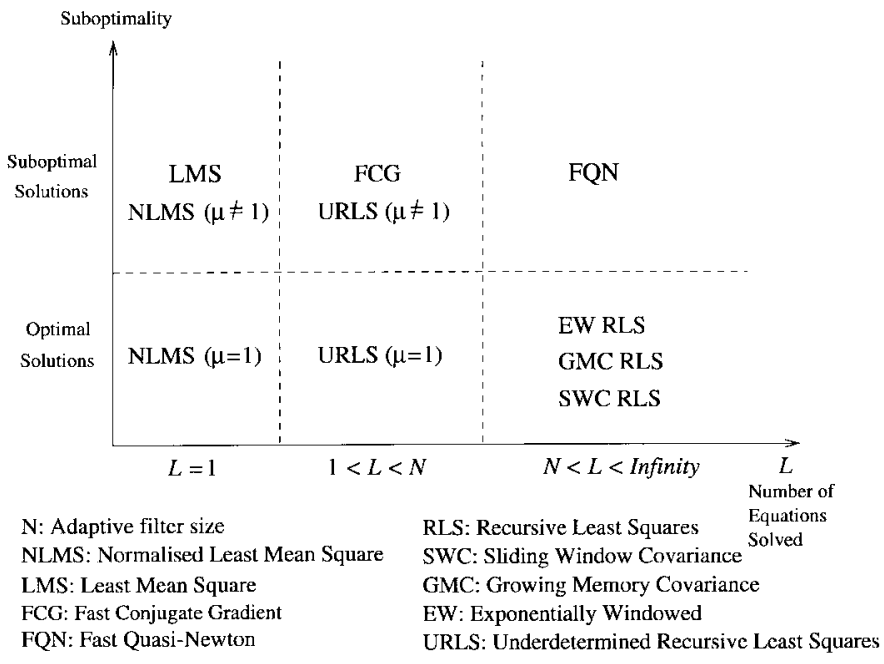

Fig. 1. Optimality comparison of adaptive algorithms within a least-square sense.

operation, but the complexity can be reduced under the assumptions that the additive noise is a white stochastic process, and the dimension of the signal subspace is known a priori [13], [15]. While the first assumption may correspond to a real situation, the second is somewhat unrealistic. The complexity of such fast algorithms is $O(N d)$, where $d$ is the dimension of the signal subspace. This computational complexity is higher than the corresponding case for the URLS family. It is reported that the subspace tracking methods suffer from slow convergence when a) the signal-to-noise-ratio (SNR) is low and $b$ ) when the dimension of the subspace assumed in the algorithm exceeds that of the true signal subspace [14].

In Section II, we introduce the URLS algorithm and derive its properties. In Section III, we continue by deriving an FTF version of the URLS algorithm, which reveals that there is an inherent whitening mechanism of order $m$, where $m$ is the number of equations to solve for $N$ unknowns $(m<$ $N)$. We refer to $m$ as the order of the URLS algorithm. Specifically, sliding window and growing window approaches are introduced where the prediction order is constant and increasing in value, respectively. The motivation to derive FTF versions lies in the fact that it possible to find implicitly fast updates of covariance and pseudo-inverse matrices using forward and backward prediction of the input signal. In addition, FTF recursions have low computational requirements. On the other hand, the FTF algorithm is notoriously unstable, but it is possible to maintain stability by adding a few equations. A less obvious approach would be the use of order recursive algorithms such as a least-squares lattice algorithm to calculate various quantities in the URLS algorithm [16], but this is beyond the scope of this paper. An alternative algorithm - the efficient URLS (EURLS) - is described in Section IV, which does not compute the adaptive filter coefficients explicitly. This reduces the computational load when the computation of the adaptive filter coefficients is not required. Approximations and relations to earlier work are discussed in Section V. Some adaptive algorithms can be shown to be approximate versions of the URLS concept. Practical issues are addressed in Section VI. It can be inferred

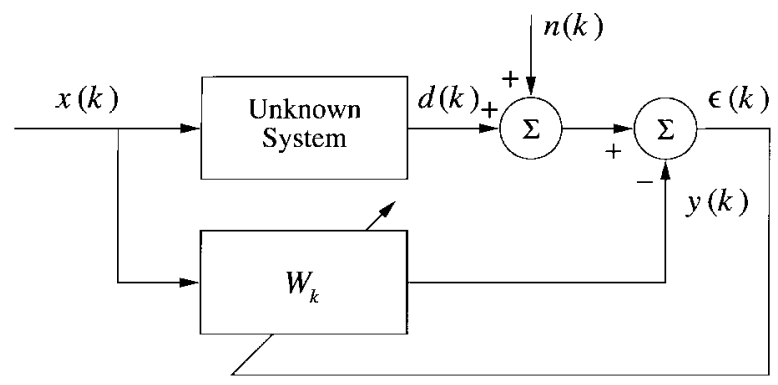

Fig. 2. System identification problem.

that utilization of the URLS family algorithms is contingent on the applicability of the matrix inversion lemma or FTF recursions, depending on whether the basic form or URLS-FTF formulation is used. If an order recursive algorithm, such as the QR-Lattice algorithm [17], was used to obtain the URLS$\mathrm{QR}$ realization, the applicability would be dependent on the feasibility of the QR-lattice implementation. We also present simulation examples and some conclusions in Sections VII and VIII.

In this paper, we assume that the adaptive filter is single channel and may have complex input signals. The notation is consistent with the earlier work of Cioffi [18]-[20] with the exception that another index is introduced both on the adaptive filter $W$ and the error vector $\mathcal{E}$ to denote the order of the URLS algorithm used in their determination. Thus, in $W_{N, m, n, k}$, we have

$N$ length of the vector

$m$ order of the URLS algorithm

$n$ window length across which the estimates of prediction variables are calculated

$k$ discrete time index.

For the sake of clarity, the unused indices will be replaced by '-' wherever applicable. Matrices, vectors, and scalars are, respectively, denoted by bold uppercase, uppercase, and lowercase letters. In scalars, the time index is also put in parentheses. The setup used in this paper is shown in Fig. 2. The input signal matrix, vector, and sample are, respectively, denoted by $\mathrm{X}, X$, and $x$. Similarly, the desired signal vector and sample are $\mathcal{D}$ and $d$, respectively. In addition, the error vector and sample are shown by $\mathcal{E}$ and $\epsilon$. The prediction filters $A, B, C$, and $D$, and the adaptive filter $W$, are assumed to be row vectors. The variables in Table I are frequently used in the sequel. The superscripts $(.)^{H},(.)^{T}$, and $(.)^{*}$ denote Hermitian transpose, ordinary transpose, and complex conjugation without transposition, respectively. We use 'complexity' to denote the number of multiplications and divisions to achieve a task.

\section{UNDERDETERMINED ORDER RECURSIVE LEAST-SQUARES ALGORITHMS}

In this section, a general framework is introduced in which some of the parameter estimation algorithms can be derived as a result of a constrained optimization (minimization) problem. The minimization problem is formulated in the light of Widrow's principle of minimal disturbance [21]. An example of this principle has appeared in [22] in the derivation 
TABLE I

PREDICTION VARIABLES

\begin{tabular}{||c||c|c||}
\hline Variables & Forward Prediction & Backward Prediction \\
\hline \hline Front Edge Residuals & $e_{m, N}(k)$ & $r_{m, N}(k)$ \\
\hline Rear Edge Residuals & $a_{m, N}(k)$ & $b_{m, N}(k)$ \\
\hline Prediction Errors & $e_{m, N-1}^{p}(k)$ & $r_{m, N-1}^{p}(k)$ \\
\hline Smoothed Errors & $a_{m, N-1}^{s}(k)$ & $b_{m, N-1}^{s}(k)$ \\
\hline Residual Powers & $\alpha_{m, N}(k)$ & $\beta_{m, N}(k)$ \\
\hline Prediction Filters & $A_{m, N, k}$ & $B_{m, N, k}$ \\
\hline Prediction Error Vectors & $E_{-, m, N, k}$ & $R_{-, m, N, k}$ \\
\hline Front Likelihood Variable & \multicolumn{2}{|c||}{$\gamma_{m, N}(k)$} \\
\hline Rear Likelihood Variable & \multicolumn{2}{|c||}{$\delta_{m, N}(k)$} \\
\hline Partial Correlation Coefficient & \multicolumn{2}{|c||}{$\rho_{m, N}(k)$} \\
\hline Correlation Coefficient & \multicolumn{2}{|c||}{$p_{n, N}(k)=X_{N, k-n}^{H} X_{N, k}$} \\
\hline Notes: $m:$ Prediction order, $N:$ Window length \\
\hline \multicolumn{2}{|c|}{}
\end{tabular}

of the NLMS algorithm. Here, it is shown that the optimization problem can be extended to cover other parameter estimation algorithms. Apart from the well-known NLMS and RLS algorithms, another class, namely, underdetermined recursive least squares algorithms, which has previously been overlooked, is considered in detail. We also show that the URLS algorithm iteratively minimizes an error criterion, the solution of which does not exist in a well-defined sense and requires the application of the pseudo-inverse (Moore-Penrose inverse) of a rectangular matrix. This is due to an underlying underdetermined set of equations for the unknown parameters. The motivation behind the URLS algorithm is to obtain a time recursive algorithm where the pseudo-inverse must be updated at each time instant as new data samples arrive.

The original URLS algorithm was proposed as early as 1984 by Ozeki [1], who named it the affine projection algorithm (AP). The concept of AP will become clear as we consider the optimization problem later in this section.

\section{A. Derivations of Adaptive Algorithms via Principle of Minimal Disturbance}

The principle of minimal disturbance states that, in the light of new input data, the parameters of an adaptive system should only be disturbed in a minimal fashion [21]. In fact, this principle is a manifestation of the optimality of leastsquares estimates but more informative in an adaptive sense as true least-squares estimates are nonadaptive. Based on this argument, the following optimization problem can be established.

Determine the tap-weight row vector of length $N$ at time $k, W_{N,-,-k}$, given the tap-input vectors $X_{N, k}, X_{N, k-1}, \ldots$, and desired responses $d(k), d(k-1), \ldots$, in order to minimize the squared Euclidean norm of the change in the tap-weight vector $W_{N,-,-, k}$

$$
\delta W_{N,-,-, k}=W_{N,-,-, k}-W_{N,-,-, k-1}
$$

subject to the constraints

$$
\begin{aligned}
W_{N,-,-, k} X_{N, k} & =d(k) \\
W_{N,-,-, k} X_{N, k-1} & =d(k-1) \\
& \vdots \\
W_{N,-,-, k} X_{N, k-m+1} & =d(k-m+1)
\end{aligned}
$$

where dashes ' - ' denote don't care quantities and $m<N$. The signals are also indicated in Fig. 2. To solve this quadratic, linearly constrained optimization problem, the method of Lagrange multipliers can be used, which yields [16]

$W_{N,-,-, k}$

$$
=W_{N,-,-, k-1}+\mathcal{E}_{m,-,-, k}^{H}\left(\mathbf{X}_{m, N, k}^{H} \mathbf{X}_{m, N, k}\right)^{-1} \mathbf{X}_{m, N, k}^{H} \text {. }
$$

The error vector $\mathcal{E}_{m,-,-, k}$ and the matrix (vector aggregate of $\left.X_{N, k}\right) \mathbf{X}_{m, N, k}$ are defined as

$$
\begin{aligned}
& \mathbf{X}_{m, N, k} \triangleq\left[\begin{array}{llll}
X_{N, k} & X_{N, k-1} & \cdots & X_{N, k-m+1}
\end{array}\right] \\
& \mathcal{E}_{m,-,-, k}^{H} \triangleq \mathcal{D}_{m, k}^{H}-W_{N,-,-, k-1} \mathbf{X}_{m, N, k}
\end{aligned}
$$

where $\mathcal{D}_{m, k}^{H}=\left[\begin{array}{lll}d(k) & \cdots & d(k-m+1)\end{array}\right]$ is the desired response vector. Equation (3) defines the $m$ th-order URLS algorithm. It can be made more general if a step-size $\mu$ is introduced to control the convergence of the algorithm, which in turn, together with the definition of the covariance matrix $\mathbf{U}_{m, N, k} \triangleq \mathbf{X}_{m, N, k}^{H} \mathbf{X}_{m, N, k}$ leads to the update equation

$$
W_{N,-,-, k}=W_{N,-,-, k-1}+\mu \mathcal{E}_{m,-,-, k}^{H} \mathbf{U}_{m, N, k}^{-1} \mathbf{X}_{m, N, k}^{H} \text {. }
$$

When $m=1$, the URLS algorithm reduces to the NLMS algorithm. Strictly speaking, the algorithm in (6) is not a least squares type algorithm unless $\mu=1$ but a combination of recursive least squares type and gradient search-type algorithms. It can be observed that the input vector at each time instant is projected onto an affine subspace that is spanned by previous input vectors subject to the condition that the matrix inverse in (6) exists. An affine subspace is not a proper linear subspace of a higher dimensional space but a hyperplane that may or may not contain the origin.

The don't care quantities in $W_{N,-,-, k}$ and $\mathcal{E}_{m,-,-, k}$ are defined next. Since the computations are evaluated over a window of length $N$ and the order is $m$, (6) can be written as

$$
W_{N, m, N, k}=W_{N, m, N, k-1}+\mu \mathcal{E}_{m, m, N, k}^{H} \mathbf{U}_{m, N, k}^{-1} \mathbf{X}_{m, N, k}^{H} .
$$

The complexity of the algorithm defined in (7) is $O(m N)+$ $O\left(m^{2}\right)$ when the matrix inversion lemma [19] is used to compute $\mathbf{U}_{m, N, k}^{-1}$ recursively. Rearrangement of (6) yields

$$
\begin{aligned}
W_{N,-,-, k}= & \mu \mathcal{D}_{m, k}^{H}\left(\mathbf{X}_{m, N, k}^{H} \mathbf{X}_{m, N, k}\right)^{-1} \mathbf{X}_{m, N, k}^{H} \\
& +W_{N,-,-, k-1}\left(\mathbf{I}_{N}-\mu \mathbf{P}_{\mathbf{X}}\right)
\end{aligned}
$$

where $\mathbf{I}_{N}$ is the $N \times N$ identity matrix, $\mathbf{P}_{\mathbf{X}}=\mathbf{X}_{m, N, k}$ $\left(\mathbf{X}_{m, N, k}^{H} \mathbf{X}_{m, N, k}\right)^{-1} \mathbf{X}_{m, N, k}^{H}$ is the projection operator onto the column space of the matrix $\mathbf{X}_{m, N, k}$, and $\mathbf{I}_{N}-\mathbf{P}_{\mathbf{X}}$ is the projection operator onto the orthogonal complement of the column space of $\mathbf{X}_{m, N, k}$ [20]. When the algorithm is optimal $(\mu=1)$, the first term on the right-hand side is the result of minimization of the following sum with respect to $W_{N,-,-, k}$

$$
\sum_{i=k-m+1}^{k}\left|d(i)-W_{N,-,-, k} X_{N, i}\right|^{2} \quad m<N .
$$

Hence, it is clear that the algorithm updates the minimumnorm solution to an RLS-like least-squares error criterion that is underdetermined: The number of equations is less than the number of unknowns. This is not a well-defined problem, and it is necessary to resort to finding the minimum-norm solution 
among several candidates and update it by projecting the last solution $W_{N,-,-, k-1}$ onto the orthogonal complement of the subspace spanned by the $m$ input vectors. In addition, the covariance matrix $\mathbf{U}$ is recursively propagated in time. Thus, the name underdetermined recursive least-squares follows. When the number of constraints is equal to the number of adaptive filter taps $(m=N)$, for $\mu=1$, the solution is $W_{N,-,-, k}=\mathcal{D}_{m, k}^{H} \mathbf{X}_{m, N, k}^{-1}$, which is the solution of the matrix equation $W \mathbf{X}=\mathcal{D}^{H}$ at every time index without using the prediction error output of the system. For $\mu \neq 1$, the second term on the right-hand side of (8) also affects the solution that calls for the use of the prediction error. When (6) is carefully examined, some adaptive algorithms that are approximations of the URLS algorithm may be identified. Some of these algorithms are described in Section V.

\section{B. Properties of the URLS Algorithm}

The key identity is the relation between the a priori and $a$ posteriori error vectors. To derive this relation, consider the a posteriori error vector

$$
\overline{\mathcal{E}}_{m, m, N, k}^{H}=\mathcal{D}_{m, k}^{H}-W_{N, m, N, k} \mathbf{X}_{m, N, k}
$$

and substitute $W_{N, m, N, k}$, which is obtained from (6). After some algebra, the desired relation is obtained as

$$
\overline{\mathcal{E}}_{m, m, N, k}^{H}=(1-\mu) \mathcal{E}_{m, m, N, k}^{H} .
$$

Therefore, the a posteriori errors are identically zero if $\mu=1$, which underscores the optimality of the least squares solution. This optimality may not be desirable in some applications, where large observation noise is present, as, for example, in acoustic echo cancellation in a hands-free telephone at low signal-to-ratio (SNR) levels [23]. High observation noise causes deviations from the optimum if $\mu$ is not chosen to be sufficiently small. Hence, the step-size may be useful to suppress the effect of the noise on the unknown system.

Order recursions of the a priori and a posteriori vectors are also needed to derive the fast algorithms presented in the following sections. To develop the order recursions, define

$$
\epsilon(i, k) \triangleq d(k)-W_{N, m, N, i} X_{N, k}
$$

so that an a priori error vector can be rewritten as

$$
\begin{aligned}
& \mathcal{E}_{m, m, N, k}^{H}=[\epsilon(k-1, k) \quad \epsilon(k-1, k-1) \\
& \cdots \quad \epsilon(k-1, k-m+1)] \text {. }
\end{aligned}
$$

As a special case of (11), the following also holds:

$$
\epsilon(i, k)=(1-\mu) \epsilon(i-1, k) \quad i=k, \ldots, k-m+1 \text {. }
$$

Consequently, the partitioned form of the a priori error vector of length $m+1$ becomes

$$
\mathcal{E}_{m+1, m, N, k}=\left[\begin{array}{c}
\mathcal{E}_{m, m, N, k} \\
\epsilon^{H}(k-1, k-m)
\end{array}\right]
$$

which is a length update relationship. Another recursion can also be found by realizing that the a priori error vector includes the lower order a posteriori error vector of the previous time instant, i.e.,

$$
\mathcal{E}_{m+1, m, N, k}=\left[\begin{array}{c}
\epsilon^{H}(k-1, k) \\
\overline{\mathcal{E}}_{m, m, N, k-1}
\end{array}\right]=\left[\begin{array}{c}
\epsilon^{H}(k-1, k) \\
(1-\mu) \mathcal{E}_{m, m, N, k-1}
\end{array}\right]
$$

which is a length-and-time update equation.

\section{URLS-FTF ALGORITHM}

Computational simplifications are possible in the URLS algorithm if the input matrix of the adaptive filter has Hankel structure [20], which permits the use of forward and backward prediction of the input signal to compute the Kalman gain without explicitly computing the inverse of the covariance matrix. In this section, we pursue the issue of updating the pseudo-inverse via FTF relations, which yield a low computational cost. On the other hand, to be of practical use, the algorithm must be stabilized as it suffers from exponential divergence of quantization errors, which stems from the hyperbolic transformation used in the downdate relation of the backward prediction filter and Kalman gain [30]. In the growing window formulation presented in Section III-B, the aforementioned hyperbolic transformation is not used; therefore, we may expect improved numerical performance. The derivation below depends on the Hilbert space approach to the development of recursive least-squares algorithms utilized in [18]-[20]. The objective is to find a fast update rule for the $\mathbf{K}_{m, N, k}^{H}=\mathbf{U}_{m, N, k}^{-1} \mathbf{X}_{m, N, k}^{H}$ matrix given in the update relation of the URLS algorithm

$$
W_{N, m, N, k}=W_{N, m, N, k-1}+\mu \mathcal{E}_{m, m, N, k}^{H} \mathbf{K}_{m, N, k}^{H} .
$$

Note that the FTF algorithm presented hereafter operates on the signal $x^{*}(k)$ rather than $x(k)$ because the definition of the matrix $\mathbf{K}$ is modified slightly such that in our development, we use $X_{N, k}=\left[\begin{array}{llll}x(k) & \cdots & x(k-N+1)\end{array}\right]^{T}$ in the definition of $\mathbf{K}$, unlike [18]-[20]. One can identify the equivalence of the $\mathbf{K}_{m, N, \boldsymbol{k}}$ matrices given in (17) and in [20], where order and time updates are presented. As in the case of all fast algorithms, the strategy is to increase the order from $m$ to $m+1$ and then decrease it back to $m$. The generic updates of $\mathbf{K}_{m, N, k}$ are derived in [20] as

$$
\begin{aligned}
& \mathbf{K}_{\mathbf{X}, \mathbf{Z}}=\left[\begin{array}{ll}
\mathbf{K}_{\mathbf{X}} & \mathbf{0}
\end{array}\right]+\mathbf{P}_{\mathbf{X}}^{\perp} \mathbf{Z}\left(\mathbf{Z}^{H} \mathbf{P}_{\mathbf{X}}^{\perp} \mathbf{Z}\right)^{-1}\left[\begin{array}{ll}
-\mathbf{Z}^{H} \mathbf{K}_{\mathbf{X}} & \mathbf{I}
\end{array}\right] \\
& \mathbf{K}_{\mathbf{Z}, \mathbf{X}}=\left[\begin{array}{ll}
\mathbf{0} & \mathbf{K}_{\mathbf{X}}
\end{array}\right]+\mathbf{P}_{\mathbf{X}}^{\perp} \mathbf{Z}\left(\mathbf{Z}^{H} \mathbf{P}_{\mathbf{X}}^{\perp} \mathbf{Z}\right)^{-1}\left[\begin{array}{ll}
\mathbf{I} & -\mathbf{Z}^{H} \mathbf{K}_{\mathbf{X}}
\end{array}\right]
\end{aligned}
$$

where $\mathbf{X}$ and $\mathbf{Z}$ are arbitrary matrices, $\mathbf{I}$ is the identity matrix, $\mathbf{X}, \mathbf{Z}$ or $\mathbf{Z}, \mathbf{X}$ in the subscript denotes an augmented matrix, $\mathbf{K}_{\mathbf{X}}^{H}=\left(\mathbf{X}^{H} \mathbf{X}\right)^{-1} \mathbf{X}^{H}, \mathbf{P}_{\mathbf{X}}=\mathbf{X}\left(\mathbf{X}^{H} \mathbf{X}\right)^{-1} \mathbf{X}^{H}$, and $\mathbf{P}_{\mathbf{X}}^{\perp}=$ $\mathbf{I}-\mathbf{P X}_{\mathbf{X}}$. In other words, (18) and (19) define pseudoinverses for new matrices $[\mathbf{X}, \mathbf{Z}]$ and $[\mathbf{Z}, \mathbf{X}]$ in terms of $\mathbf{K}_{\mathbf{X}}$. In our case, when $\mathbf{X}=\mathbf{X}_{m, N, k}$ and $\mathbf{Z}=X_{N, k-m}$ are substituted in (18), the following order update recursion is obtained:

$$
\mathbf{K}_{m+1, N, k}^{H}=\left[\begin{array}{c}
\mathbf{K}_{m, N, k}^{H} \\
0
\end{array}\right]+\frac{1}{\beta_{m, N}(k)} B_{m, N, k}^{H} R_{N, m, N, k}^{H}
$$

where $\beta_{m, N}(k)=Z^{H} \mathbf{P}_{\mathbf{X}}^{\perp} Z, B_{m, N, k}=\left[-Z^{H} \mathbf{K}_{\mathbf{X}} 1\right]$ and $R_{N, m, N, k}=\mathbf{P}_{\mathbf{X}}^{\perp} Z$. To obtain the other recursion substitute 
$\mathbf{X}=\mathbf{X}_{m, N, k-1}$ and $\mathbf{Z}=X_{N, k}$ in (19), which yields an order-and-time update

$$
\mathbf{K}_{m+1, N, k}^{H}=\left[\begin{array}{c}
0 \\
\mathbf{K}_{m, N, k-1}^{H}
\end{array}\right]+\frac{1}{\alpha_{m, N}(k)} A_{m, N, k}^{H} E_{N, m, N, k}^{H}
$$

where $\alpha_{m, N}(k)=Z^{H} \mathbf{P}_{\overline{\mathbf{X}}}^{\perp} Z, A_{m, N, k}=\left[\begin{array}{ll}1 & -Z^{H} \mathbf{K}_{\mathbf{X}}\end{array}\right]$ and $E_{N, m, N, k}=\mathbf{P}_{\mathbf{X}}^{\frac{1}{X}} Z$. It is also convenient to define

$$
G_{N, m, N, k} \triangleq \mu \mathcal{E}_{m, m, N, k}^{H} \mathbf{K}_{m, N, k}^{H}
$$

where the subscripts are similar to $W$. Then, two updates for $G_{N, m+1, N, k}$ may be obtained by, respectively, multiplying the partitioned forms of the a priori error vector in (15) and (20) with (20) and (21)

$$
\begin{aligned}
& G_{N, m+1, N, k} \\
& \quad=\mu\left(\frac{1}{\mu} G_{N, m, N, k}+\frac{1}{\beta_{m, N}(k)} \eta_{m, N}(k) R_{N, m, N, k}^{H}\right) \\
& G_{N, m+1, N, k} \\
& \quad=\mu\left(\frac{1-\mu}{\mu} G_{N, m, N, k-1}+\frac{1}{\alpha_{m, N}(k)} \zeta_{m, N}(k) E_{N, m, N, k}^{H}\right)
\end{aligned}
$$

where the inner products $\eta_{m, N}(k)$ and $\zeta_{m, N}(k)$ are

$$
\begin{aligned}
& \eta_{m, N}(k) \triangleq \mathcal{E}_{m+1, m, N, k}^{H} B_{m, N, k}^{H}, \\
& \zeta_{m, N}(k) \triangleq \mathcal{E}_{m+1, m, N, k}^{H} A_{m, N, k}^{H} .
\end{aligned}
$$

Hence, the recursion for $G$ is obtained by equating (23a) and (23b)

$$
\begin{aligned}
G_{N, m, N, k}= & (1-\mu) G_{N, m, N, k-1} \\
& +\mu \frac{1}{\alpha_{m, N}(k)} \zeta_{m, N}(k) E_{N, m, N, k}^{H} \\
& -\mu \frac{1}{\beta_{m, N}(k)} \eta_{m, N}(k) R_{N, m, N, k}^{H}
\end{aligned}
$$

which, with the relation

$$
W_{N, m, N, k}=W_{N, m, N, k-1}+G_{N, m, N, k}
$$

completes the derivation of the URLS-FTF algorithm.

Equation (25) defines the update relation of the $G_{N, m, N, k}$ vector in the URLS algorithm. The appearance of the prediction filters indicates that whitening is performed on the input signal, i.e., the input signal is decorrelated prior to filtering. The important point is that the effective prediction order of the input is $m-1$, which can be seen by the following example: The NLMS algorithm corresponds to the zerothorder prediction of the input because it uses the input signal itself in the update. It also corresponds to the URLS algorithm with $m=1$, although the URLS-FTF algorithm uses firstorder prediction of the input to realize the NLMS algorithm, which is obviously unnecessary. Thus, the URLS algorithm uses prediction filters of the order one greater than the effective prediction order of the input, except when $\mu=1$, which enables the use of prediction filters at the same order as the effective prediction order of the input, as discussed in Section V-C. We can say that the URLS-FTF formulation is useful for $m>1$. The fact that $m$ is larger than the effective prediction order of the input is due to the update strategy of increasing and decreasing the order, i.e., $m \rightarrow m+1 \rightarrow m$ to find a fast update. Many variations of the algorithm can be found, depending on how the prediction error vectors $E_{N, m, N, k}$ and $R_{N, m, N, k}$ are computed. In the sections below, sliding window and growing window forms of the URLS-FTF algorithm are considered.

\section{A. URLS-FTF Sliding Window Covariance (URLS-FTF-SWC) Algorithm}

The fast and exact method to carry out the computations of the URLS algorithm is to treat the prediction problem as a sliding window covariance RLS algorithm with order $m$ and window length $N$. The covariance matrix is estimated over a window of length $N$ (c.f. (6)). We need to consider the following updates of the transversal filters in the sliding window covariance FTF (FTF-SWC) algorithm [19]

$$
\begin{aligned}
& {\left[\begin{array}{c}
A_{m, N+1, k} \\
\tilde{C}_{m+1, N+1, k}
\end{array}\right]=\mathbf{T}_{1, k}\left[\begin{array}{cc}
A_{m, N, k-1} \\
{\left[\begin{array}{cc}
0 & \tilde{C}_{m, N+1, k-1}
\end{array}\right]}
\end{array}\right]} \\
& {\left[\begin{array}{c}
B_{m, N+1, k} \\
{\left[\tilde{C}_{m, N+1, k} 0\right]}
\end{array}\right]=\mathbf{T}_{2, k}\left[\begin{array}{c}
B_{m, N, k-1} \\
\tilde{C}_{m+1, N+1, k}
\end{array}\right]} \\
& {\left[\begin{array}{c}
A_{m, N, k} \\
D_{m+1, N+1, k}
\end{array}\right]=\mathbf{T}_{3, k}\left[\begin{array}{cc}
A_{m, N+1, k} \\
0 & D_{m, N+1, k-1}
\end{array}\right]}
\end{aligned}
$$

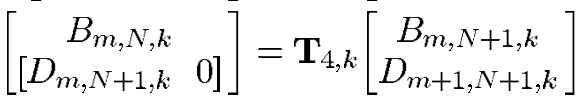

where

$$
\begin{aligned}
\mathbf{T}_{1, k} & =\left[\begin{array}{cc}
1 & e_{m, N+1}(k) \\
-\frac{e_{m, N}^{p H}(k)}{\alpha_{m, N}(k-1)} & 1
\end{array}\right] \\
\mathbf{T}_{2, k} & =\left[\begin{array}{cc}
1-r_{m, N+1}(k) \tilde{C}_{m+1, N+1, k}^{(m)} & r_{m, N+1}(k) \\
-\tilde{C}_{m+1, N+1, k}^{(m)} & 1
\end{array}\right] \\
\mathbf{T}_{3, k} & =\left[\begin{array}{cc}
1 & -a_{m, N}^{s}(k) \\
-\frac{a_{m, N+1}^{H}(k)}{\alpha_{m, N+1}(k)} & 1
\end{array}\right] \\
\mathbf{T}_{4, k} & =\left[\begin{array}{cc}
1+b_{m, N}^{s}(k) D_{m+1, N+1, k}^{(m)} & -b_{m, N}^{s}(k) \\
-D_{m+1, N+1, k}^{(m)} & 1
\end{array}\right]
\end{aligned}
$$

and $\tilde{C}$ and $D$ are used for forward and backward pinning estimation [19]. $\tilde{C}$ is also known as the dual Kalman gain vector. In addition, the prediction error vectors are defined as

$$
\begin{aligned}
V_{N, m+1, N+1, k}^{H} & \triangleq \tilde{C}_{m+1, N+1, k} \mathbf{X}_{m+1, N, k}^{H}, \\
Y_{N, m+1, N+1, k}^{H} & \triangleq D_{m+1, N+1, k} \mathbf{X}_{m+1, N, k}^{H}, \\
E_{N, m, N+1, k}^{H} & \triangleq A_{m, N+1, k} \mathbf{X}_{m+1, N, k}^{H}, \\
R_{N, m, N+1, k}^{H} & \triangleq B_{m, N+1, k} \mathbf{X}_{m+1, N, k}^{H} .
\end{aligned}
$$

Some useful results obtained from the above definitions are

$$
\begin{aligned}
V_{N, m, N+1, k-1}^{H} & =\left[\begin{array}{ll}
0 & \tilde{C}_{m, N+1, k-1}
\end{array}\right] \mathbf{X}_{m+1, N, k}^{H}, \\
V_{N, m, N+1, k}^{H} & =\left[\begin{array}{ll}
\tilde{C}_{m, N+1, k} & 0
\end{array}\right] \mathbf{X}_{m+1, N, k}^{H} \\
Y_{N, m, N+1, k-1}^{H} & =\left[\begin{array}{ll}
0 & D_{m, N+1, k-1}
\end{array}\right] \mathbf{X}_{m+1, N, k}^{H} \\
Y_{N, m, N+1, k}^{H} & =\left[\begin{array}{ll}
D_{m, N+1, k} & 0
\end{array}\right] \mathbf{X}_{m+1, N, k}^{H}, \\
E_{N-1, m, N, k-1}^{H} & =A_{m, N, k-1} \mathbf{X}_{m+1, N-1, k-1}^{H}
\end{aligned}
$$


TABLE II

URLS-FTF-SWC ALGORITHM

\begin{tabular}{|c|c|}
\hline Computation & Complexity \\
\hline \multicolumn{2}{|l|}{ Prediction } \\
\hline \multicolumn{2}{|l|}{$\begin{array}{l}\text { Prediction parts of the front and rear edges of the window in the sliding } \\
\text { window FTF algorithm of order } m \text { and window length } N \text { for } x^{*}(k) \text {, }\end{array}$} \\
\hline 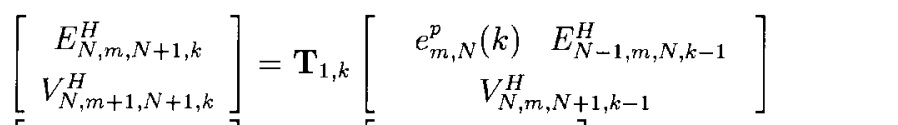 & $2 N$ \\
\hline$\left[\begin{array}{c}E_{N, m, N, k}^{H} \\
Y_{N, m+1, N+1, k}^{H}\end{array}\right]=\mathbf{T}_{3, k}\left[\begin{array}{c}E_{N, m, N+1, k}^{H} \\
Y_{N, m, N+1, k-1}^{H}\end{array}\right]$ & $2 N$ \\
\hline$\left[\begin{array}{l}R_{N, m, N+1, k}^{H} \\
V_{N, m, N+1, k}^{H}\end{array}\right]=\mathbf{T}_{2, k}\left[\begin{array}{cc}r_{m, N}^{p}(k) & R_{N-1, m, N, k-1}^{H} \\
V_{N, m+1, N+1, k}^{H}\end{array}\right.$ & $3 N$ \\
\hline$\left[\begin{array}{c}R_{N, m, N, k}^{H} \\
Y_{N, m, N+1, k}^{H}\end{array}\right]=\mathbf{T}_{\mathbf{4}, k}\left[\begin{array}{c}R_{N, m, N+1, k}^{H} \\
Y_{N, m+1, N+1, k}^{H}\end{array}\right]$ & $3 N$ \\
\hline Total (Prediction) & $10 N+10 m+22(4 \mathrm{~d}$ \\
\hline \multicolumn{2}{|l|}{ Joint Process Extension } \\
\hline$\epsilon_{m, N}^{p}(k)=d(k)-W_{N, m, N, k-1} X_{N, k}$ & $N$ \\
\hline $\mathcal{E}_{m+1, m, N, k}^{H}=\left[\begin{array}{ll}\epsilon_{m, N}^{p}(k) & (1-\mu) \mathcal{E}_{m, m, N, k-1}^{H}\end{array}\right]$ & $m$ \\
\hline$\eta_{m, N}(k)=\mathcal{E}_{m+1, m, N, k}^{H} B_{m, N, k}^{H}$ & $m$ \\
\hline$\zeta_{m, N}(k)=\mathcal{E}_{m+1, m, N, k}^{H} A_{m, N, k}^{H}$ & $m$ \\
\hline$G_{N, m, N, k}=(1-\mu) G_{N, m, N, k-1}+\mu \frac{\zeta_{m, N}(k)}{\alpha_{m, N}(k)} E_{N, m, N, k}^{H}$ & $3 N+2$ (2 divides $)$ \\
\hline$W_{N, m, N, k}=W_{N, m, N, k-1}+G_{N, m, N, k}$ & - \\
\hline Total (Prediction and Joint Process Estimation) & $14 N+13 m+24$ (6 divides) \\
\hline
\end{tabular}

$$
\begin{aligned}
R_{N-1, m, N, k-1}^{H} & =B_{m, N, k-1} \mathbf{X}_{m+1, N-1, k-1}^{H} \\
E_{N, m, N, k}^{H} & =A_{m, N, k} \mathbf{X}_{m+1, N, k}^{H} \\
R_{N, m, N, k}^{H} & =B_{m, N, k} \mathbf{X}_{m+1, N, k}^{H} \\
E_{N, m,-, k-1}^{H} & =\left[\begin{array}{ll}
E_{N-1, m,-, k-1}^{H} & A_{m,-, k-1} X_{m+1, k-N}^{*}
\end{array}\right] .
\end{aligned}
$$

When both sides of (27a)-(27b) and (27c)-(27d) are multiplied, respectively, by $\left[\begin{array}{lll}X_{m+1, k}^{*} & \left.\mathbf{X}_{m+1, N-1, k-1}^{H}\right] \text { and }\end{array}\right.$ $\mathbf{X}_{m+1, N, k}^{H}$, the following updates

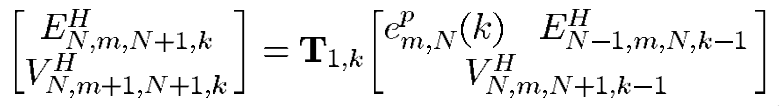

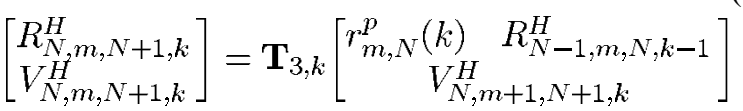

and downdates

$$
\begin{aligned}
{\left[\begin{array}{c}
E_{N, m, N, k}^{H} \\
Y_{N, m+1, N+1, k}^{H}
\end{array}\right] } & =\mathbf{T}_{2, k}\left[\begin{array}{c}
E_{N, m, N+1, k}^{H} \\
Y_{N, m, N+1, k-1}^{H}
\end{array}\right] \\
{\left[\begin{array}{c}
R_{N, m, N, k}^{H} \\
Y_{N, m, N+1, k}^{H}
\end{array}\right] } & =\mathbf{T}_{4, k}\left[\begin{array}{c}
R_{N, m, N+1, k}^{H} \\
Y_{N, m+1, N+1, k}^{H}
\end{array}\right]
\end{aligned}
$$

of the prediction error vectors can be found.
The derivation shows that the prediction error vectors $E_{N, m, N, k-1}$ and $R_{N, m, N, k-1}$ can be updated using intermediate vectors $Y, V$, and transformations $\mathbf{T}_{1, k}, \ldots, \mathbf{T}_{4, k}$, which together represent an overall complexity of $O(N)+O(m)$. The complete algorithm is presented in Table II.

\section{B. URLS-FTF Growing Window Covariance (URLS-FTF-GWC) Algorithm}

If it is desired to increase the size of the prediction in time rather than keeping it fixed as in the case of the sliding window formulation, we only use the order-and-time update of (23b). In this case, the prediction size is incremented as (23a) is dropped, which was previously employed to keep the prediction size fixed. Hence

$$
\begin{aligned}
G_{N, m+1, N, k}= & (1-\mu) G_{N, m, N, k-1} \\
& +\mu \frac{1}{\alpha_{m, N}(k)} \zeta_{m, N}(k) E_{N, m, N, k}^{H}
\end{aligned}
$$

and this equation together with the update

$$
W_{N, m+1, N, k}=W_{N, m, N, k-1}+G_{N, m+1, N, k}
$$

defines the URLS-FTF-GWC algorithm. In this algorithm, order updates are also required for the prediction error filters $A$ and $B$ and the associated prediction error vectors. In particular, 
the generalized Levinson recursions [18] provide these updates as follows:

$$
\begin{aligned}
{\left[\begin{array}{c}
A_{m, N+1, k} \\
\tilde{C}_{m+1, N+1, k}
\end{array}\right] } & =\mathbf{T}_{1, k}\left[\begin{array}{cc} 
& A_{m, N, k-1} \\
0 & \tilde{C}_{m, N+1, k-1}
\end{array}\right] \\
{\left[\begin{array}{c}
A_{m, N, k} \\
D_{m+1, N+1, k}
\end{array}\right] } & =\mathbf{T}_{3, k}\left[\begin{array}{cc} 
& A_{m, N+1, k} \\
0 & D_{m, N+1, k-1}
\end{array}\right] \\
{\left[\begin{array}{c}
A_{m+1, N, k} \\
B_{m+1, N, k}
\end{array}\right] } & =\mathbf{T}_{5, k}\left[\begin{array}{cc}
{\left[\begin{array}{cc}
A_{m, N, k} & 0
\end{array}\right]} \\
0 & B_{m, N, k-1}
\end{array}\right]
\end{aligned}
$$

where

$$
\mathbf{T}_{\tilde{\delta}, k}=\left[\begin{array}{cc}
1 & -\frac{\rho_{m, N}(k)}{\beta_{m, L}(k-1)} \\
-\frac{\rho_{m, N}^{H}(k)}{\alpha_{m, L}(k)} & 1
\end{array}\right] .
$$

The vector definitions $V, Y, E$, and $R$ of (29a)-(29d) also hold. When both sides of (45a), (45b), and (45c) are, respectively, multiplied by $\left[X_{m+1, k}^{*} \quad \mathbf{X}_{m+1, N-1, k-1}^{H}\right], \mathbf{X}_{m+1, N, k}^{H}$, and $\mathbf{X}_{m+2, N, k}^{H}$, the following updates and downdates of the prediction error vectors are obtained:

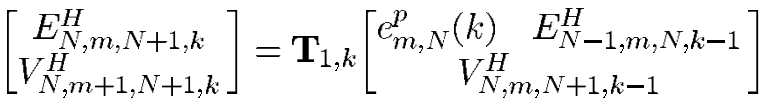

$$
\begin{aligned}
& {\left[\begin{array}{c}
E_{N, m, N, k}^{H} \\
Y_{N, m+1, N+1, k}^{H}
\end{array}\right]=\mathbf{T}_{3, k}\left[\begin{array}{c}
E_{N, m, N+1, k}^{H} \\
Y_{N, m, N+1, k-1}^{H}
\end{array}\right]} \\
& {\left[\begin{array}{l}
E_{N, m+1, N, k}^{H} \\
R_{N, m+1, N, k}^{H}
\end{array}\right]=\mathbf{T}_{5, k}\left[\begin{array}{c}
E_{N, m, N, k}^{H} \\
R_{N, m, N, k-1}^{H}
\end{array}\right] \text {. }}
\end{aligned}
$$

We also need to establish the order recursions for the residual powers using the relations in [18]

$$
\begin{aligned}
\alpha_{m+1, N}(k)= & \alpha_{m, N}(k)-\rho_{m, N}(k) \beta_{m, N}^{-1}(k-1) \rho_{m, N}^{H}(k) \\
\beta_{m+1, N}(k)= & \beta_{m, N}(k-1)-\rho_{m, N}^{H}(k) \alpha_{m, N}^{-1}(k) \rho_{m, N}(k) \\
\gamma_{m+1, N+1}(k)= & \gamma_{m, N+1}(k-1) \\
& -e_{m, N+1}^{H}(k) \alpha_{m, N+1}^{-1}(k) e_{m, N+1}(k) \\
\delta_{m+1, N+1}(k)= & \delta_{m, N+1}(k-1) \\
& -a_{m, N+1}^{H}(k) \alpha_{m, N+1}^{-1}(k) a_{m, N+1}(k) .
\end{aligned}
$$

Equation (50) is not a time update equation. In order to find a time update for $\alpha$, the following two-stage procedure may be adopted

$$
\begin{aligned}
\alpha_{m, N+1}(k) & =\alpha_{m, N}(k-1)+e_{m, N}^{p}(k) e_{m, N+1}^{H}(k), \\
\alpha_{m, N}(k) & =\alpha_{m, N+1}(k)-a_{m, N}^{s}(k) a_{m, N+1}^{H}(k) .
\end{aligned}
$$

The complete algorithm can be found in Table III, where $k_{s}$ refers to the time instant that the GWC recursions starts. It makes the parameter $m$ time-varying, i.e., $m$ is incremented by one at each instant $k>k_{s}$.

In order to exploit fully the potential of the combination of the sliding window and growing window fast URLS algorithms, criteria are required to sense the need for prediction order change, which requires further research. Some earlier reported criteria can be used as in [24] (although it was developed in a different context) that detect the need for change in the window length for a lattice least-squares algorithm.

\section{Normalized URLS-FTF Algorithms}

The algorithms of the previous sections can be modified to employ normalized forward and backward prediction errors to enhance the numerical behavior of the FTF family. The normalized prediction error filters and vectors are defined as [19], [25]

$$
\begin{aligned}
& \bar{A}_{m, N, k} \triangleq \frac{A_{m, N, k}}{\alpha_{m, N}^{1 / 2}(k)} \\
& \bar{B}_{m, N, k} \triangleq \frac{B_{m, N, k}}{\beta_{m, N}^{1 / 2}(k)} \\
& \bar{E}_{N, m, N, k} \triangleq \frac{E_{N, m, N, k}}{\alpha_{m, N}^{1 / 2}(k)} \\
& \bar{R}_{N, m, N, k} \triangleq \frac{R_{N, m, N, k}}{\beta_{m, N}^{1 / 2}(k)} .
\end{aligned}
$$

Using the definitions of $\zeta_{m, N}(k)$ and $\eta_{m, N}(k)$ in (24) and rewriting $\alpha_{m, N}(k)$ and $\beta_{m, N}(k)$ as the product of their square roots, the update of $G_{N, m, N, k}$ in (25) can be written in terms of the normalized quantities

$$
\begin{aligned}
G_{N, m, N, k}= & (1-\mu) G_{N, m, N, k-1}+\mu \bar{\zeta}_{m, N}(k) \bar{E}_{N, m, N, k}^{H} \\
& -\mu \bar{\eta}_{m, N}(k) \bar{R}_{N, m, N, k}^{H}
\end{aligned}
$$

where

$$
\begin{aligned}
& \bar{\zeta}_{m, N}(k) \triangleq \mathcal{E}_{m+1, m, N, k}^{H} \bar{A}_{m, N, k}^{H} \\
& \bar{\eta}_{m, N}(k) \triangleq \mathcal{E}_{m+1, m, N, k}^{H} \bar{B}_{m, N, k}^{H} .
\end{aligned}
$$

The above approach can be extended to cover the growing window covariance form as well. For example, the update of $G_{N, m, N, k}$ in (56) becomes

$$
G_{N, m+1, N, k}=(1-\mu) G_{N, m, N, k-1}+\mu \bar{\zeta}_{m, N}(k) \bar{E}_{N, m, N, k}^{H} .
$$

\section{EFFICIENT IMPLEMENTATION OF THE URLS ALGORITHM}

In this section, a complexity reduction procedure is introduced for the URLS algorithm. The complexity is reduced to $2 N+9 m-1$ (two divides) in the joint process estimation. We refer to the resulting formulation as the efficient URLS (EURLS) algorithm. In the derivation below, $\Upsilon_{m, N, k}^{(i)}$ refers to the $i$ th entry of the $m$-element vector $\Upsilon$ estimated over $N$ samples. Let us start by considering the update of the URLS algorithm

$$
W_{N, m, N, k}=W_{N, m, N, k-1}+\mu \underbrace{\mathcal{E}_{m, m, N, k}^{H} \mathbf{U}_{m, N, k}^{-1}}_{\Upsilon_{m, N, k}} \mathbf{X}_{m, N, k}^{H} .
$$

When (59) is rewritten in terms of the individual elements of the $\Upsilon_{m, N, k}$ vector and $\mathbf{X}_{m, N, k}$ matrix, we obtain

$$
\begin{aligned}
W_{N, m, N, k}=W_{N, m, N, k-1}+\mu[ & \Upsilon_{m, N, k}^{(1)} X_{N, k}^{H}+\cdots \\
& \left.+\Upsilon_{m, N, k}^{(m)} X_{N, k-m+1}^{H}\right] .
\end{aligned}
$$

Now, define the vector $S_{1, k-1}$ such that

$$
\begin{aligned}
S_{1, k-1} \triangleq W_{N, m, N, k-1}-\mu & {\left[\Upsilon_{m, N, k-1}^{(1)} X_{N, k-1}^{H}+\cdots\right.} \\
& \left.+\Upsilon_{m, N, k-1}^{(m-1)} X_{N, k-m+1}^{H}\right]
\end{aligned}
$$

where only the time index is retained for the sake of clarity. 
Rewriting $S_{1, k-1}$ at time $k$,

$S_{1, k}=W_{N, m, N, k}-\mu\left[\Upsilon_{m, N, k}^{(1)} X_{N, k}^{H}+\cdots+\Upsilon_{m, N, k}^{(m-1)} X_{N, k-m+2}^{H}\right]$

we can obtain $W_{N, m, N, k-1}$, in terms of $S_{1, k-1}$

$$
\begin{aligned}
W_{N, m, N, k-1}=S_{1, k-1}+\mu[ & \Upsilon_{m, N, k-1}^{(1)} X_{N, k-1}^{H}+\cdots \\
& \left.+\Upsilon_{m, N, k-1}^{(m-1)} X_{N, k-m+1}^{H}\right]
\end{aligned}
$$

which is equal to, from (60)

$$
\begin{aligned}
W_{N, m, N, k-1}=W_{N, m, N, k}-\mu & {\left[\Upsilon_{m, N, k}^{(1)} X_{N, k}^{H}+\cdots\right.} \\
& \left.+\Upsilon_{m, N, k}^{(m)} X_{N, k-m+1}^{H}\right] .
\end{aligned}
$$

In (64), the term $S_{1, k}$ can be identified as

$$
W_{N, m, N, k-1}=S_{1, k}-\mu \Upsilon_{m, N, k}^{(m)} X_{N, k-m+1}^{H} \text {. }
$$

By comparing (63) and (65), a recursion in terms of $S_{1, k}$ is obtained

$$
\begin{aligned}
S_{1, k}= & S_{1, k-1}+\mu\left[\Upsilon_{m, N, k-1}^{(1)} X_{N, k-1}^{H}+\cdots\right. \\
& \left.+\Upsilon_{m, N, k-1}^{(m-2)} X_{N, k-m+2}^{H}\right] \\
+\mu\left[\Upsilon_{m, N, k}^{(m)}\right. & \left.+\Upsilon_{m, N, k-1}^{(m-1)}\right] X_{N, k-m+1}^{H}
\end{aligned}
$$

The next step is to define a new set of coefficients $\Omega_{1, k}$ such that

$$
\begin{aligned}
& \Omega_{1, k}^{(m-1)} \triangleq \Upsilon_{m, N, k}^{(m)}+\Upsilon_{m, N, k-1}^{(m-1)} \\
& \Omega_{1, k}^{(m-2)} \triangleq \Upsilon_{m, N, k-1}^{(m-2)} \\
& \vdots \\
& \Omega_{1, k}^{(1)} \triangleq \Upsilon_{m, N, k-1}^{(1)}
\end{aligned}
$$

where only the vector number index is retained for the sake of simplicity. The number of entries in the $\Omega$ vectors can easily be inferred from the vector number, for example, $\Omega_{i, k}$ has $m-i$ elements. Thus, (66) becomes

$$
\begin{aligned}
S_{1, k}=S_{1, k-1}+\mu & {\left[\Omega_{1, k}^{(1)} X_{N, k-1}^{H}+\cdots+\Omega_{1, k}^{(m-2)} X_{N, k-m+2}^{H}\right.} \\
& \left.+\Omega_{1, k}^{(m-1)} X_{N, k-m+1}^{H}\right] .
\end{aligned}
$$

Another recursion that is similar to (60) has been obtained, and the same procedure can be applied. After iterating in the same fashion, the last recursion that may be obtained is

$$
S_{m-2, k}=S_{m-2, k-1}+\mu\left[\Omega_{m-2, k}^{(2)}+\Omega_{m-2, k-1}^{(1)}\right] X_{N, k-m+1}^{H} \text {. }
$$

Note that

$$
\begin{aligned}
\Omega_{m-2, k}^{(2)} & =\Upsilon_{m, N, k}^{(m)}+\Upsilon_{m, N, k-1}^{(m-1)}+\cdots+\Upsilon_{m, N, k-m+2}^{(2)} \\
\Omega_{m-2, k-1}^{(1)} & =\Upsilon_{m, N, k-m+1}^{(1)}
\end{aligned}
$$

which leads to

$$
\begin{aligned}
S_{m-1, k}=S_{m-1, k-1}+\mu[ & \Upsilon_{m, N, k}^{(m)}+\Upsilon_{m, N, k-1}^{(m-1)}+\cdots \\
& \left.+\Upsilon_{m, N, k-m+1}^{(1)}\right] X_{N, k-m+1}^{H} .
\end{aligned}
$$

Therefore, by iterating on $S_{m-1, k}$ in time, the number of multiplications can be reduced from $m N$ to $N$. The adaptive filter output $W_{N, m, N, k-1} X_{N, k}$ in terms of $S_{m-1, k}$ remains to be calculated. To obtain this expression, we use the recursive definition of $S_{m-1, k}$, which yields

$S_{m-1, k-1} X_{N, k}$

$$
\begin{aligned}
& =W_{N, m, N, k-1} X_{N, k} \\
& \quad-\mu\left[\Upsilon_{m, N, k-1}^{(1)} X_{N, k-1}^{H}+\cdots+\Upsilon_{m, N, k-1}^{(m-1)} X_{N, k-m+1}^{H}\right] X_{N, k} \\
& -\mu\left[\Omega_{1, k-1}^{(1)} X_{N, k-2}^{H}+\cdots+\Omega_{1, k-1}^{(m-2)} X_{N, k-m+1}^{H}\right] X_{N, k} \\
& \quad \vdots \\
& \quad-\mu\left[\Omega_{m-3, k-1}^{(1)} X_{N, k-m+2}^{H}+\Omega_{m-3, k-1}^{(2)} X_{N, k-m+1}^{H}\right] X_{N, k} \\
& -\mu \Omega_{m-2, k-1}^{(1)} X_{N, k-m+1}^{H} X_{N, k} .
\end{aligned}
$$

The above equation can be written in terms of the crossproducts of the input signal (Table I) and $\Upsilon_{m, N, k}$ vector so that

$$
\begin{aligned}
S_{m-1, k-1} & X_{N, k} \\
= & W_{N, m, N, k-1} X_{N, k} \\
& -\mu\left[\Upsilon_{m, N, k-1}^{(m-1)}+\cdots+\Upsilon_{m, N, k-m+1}^{(1)}\right] p_{m-1, N}(k) \\
& \vdots \\
& -\mu\left[\Upsilon_{m, N, k-1}^{(2)}+\Upsilon_{m, N, k-2}^{(1)}\right] p_{2, N}(k) \\
& -\mu \Upsilon_{m, N, k-1}^{(1)} p_{1, N}(k) \\
= & W_{N, m, N, k-1} X_{N, k}-\mu \Phi_{m-1, k-1} P_{m-1, N, k}
\end{aligned}
$$

where $P$ is the column vector of crossproducts, and the elements of the row vector $\Phi$ are the combinations of the elements of the $\Upsilon$ vector. Hence, $\epsilon_{m, N}^{p}(k)=W_{N, m, N, k-1} X_{N, k}$ can be computed from (74), requiring $2 m-2$ operations to compute the crossproducts and $m-1$ operations to multiply $\Phi_{m-1, k-1}$ by the crossproducts. The overall complexity in this part becomes $3 m-3$. The update rule for the $\Phi$ vector is seen to be

$$
\left[\Phi_{m-1, k} \quad \phi(k)\right]=\Upsilon_{m, N, k}+\left[\begin{array}{ll}
0 & \Phi_{m-1, k-1}
\end{array}\right] .
$$

Using this, (72) can be rewritten as

$$
S_{m-1, k}=S_{m-1, k-1}+\mu \phi(k) X_{N, k-m+1}^{H} .
$$

A fast update rule can also be found for the $\Upsilon_{m, N, k}$ vector. We use the following order and time updates and downdates of the prediction error vector (see Section II) and the covariance matrix [20]

$$
\mathbf{U}_{m+1, N, k}^{-1}=\left[\begin{array}{cc}
\mathbf{U}_{m, N, k}^{-1} & 0 \\
0^{H} & 0
\end{array}\right]+\frac{1}{\beta_{m, N}(k)} B_{m, N, k}^{H} B_{m, N, k}
$$

$$
\mathbf{U}_{m+1, N, k}^{-1}=\left[\begin{array}{cc}
0 & 0^{H} \\
0 & \mathbf{U}_{m, N, k-1}^{-1}
\end{array}\right]+\frac{1}{\alpha_{m, N}(k)} A_{m, N, k}^{H} A_{m, N, k}
$$

$\mathcal{E}_{m+1, m, N, k}^{H}=\left[\begin{array}{ll}\mathcal{E}_{m, m, N, k}^{H} & \epsilon(k-1, k-m)\end{array}\right]$

$$
\mathcal{E}_{m+1, m, N, k}^{H}=\left[\begin{array}{ll}
\epsilon(k-1, k) & (1-\mu) \mathcal{E}_{m, m, N, k-1}^{H}
\end{array}\right]
$$

where $\epsilon(k-1, k)=\epsilon_{m, N}^{p}(k)=d(k)-W_{N, m, N, k-1} X_{N, k}$. When (77a) and (77b) are multiplied, respectively, with (78a) and (78b), we obtain

$$
\begin{aligned}
& \Upsilon_{m+1, N, k}=\left[\begin{array}{ll}
\Upsilon_{m, N, k} & 0
\end{array}\right]+\frac{1}{\beta_{m, N}(k)} \eta_{m, N}(k) B_{m, N, k} \\
& =\left[\begin{array}{ll}
0 & (1-\mu) \Upsilon_{m, N, k-1}
\end{array}\right] \\
& +\frac{1}{\alpha_{m, N}(k)} \zeta_{m, N}(k) A_{m, N, k}
\end{aligned}
$$


TABLE III

URLS-FTF-GWC ALGORITHM

\begin{tabular}{|c|c|}
\hline Computation & Complexity \\
\hline \multicolumn{2}{|l|}{$\begin{array}{l}\text { Prediction } \\
\text { (From Table 1) } P_{m}^{H}\end{array}$} \\
\hline$P_{m+1, N, k}=P_{m+1, N, k-1}+x(k) X_{m+1, k-1}^{*}-x(k-N) X_{m+1, k-N-1}^{*}$ & $2 m+2$ \\
\hline$\rho_{m, N}(k)=P_{m+1, N, k}^{H} B_{m, N, k-1}^{H}$ & $m$ \\
\hline$e_{m, N}^{p}(k)=A_{m, N, k-1} X_{m+1, k}^{*}$ & $m$ \\
\hline$e_{m, N+1}(k)=e_{m, N}^{p}(k) \gamma_{m, N+1}(k-1)$ & 1 \\
\hline$A_{m, N+1, k}=A_{m, N, k-1}+e_{m, N+1}(k)\left[\begin{array}{ll}0 & \tilde{C}_{m, N+1, k-1}\end{array}\right]$ & $m$ \\
\hline$a_{m, N+1}(k)=A_{m, N+1, k} X_{m+1, k-N}^{*}$ & $m$ \\
\hline$a_{m, N}^{s}(k)=\frac{a_{m, N+1}(k)}{\delta_{m, N+1}(k-1)}$ & (1 divide) \\
\hline$\alpha_{m, N+1}(k)=\alpha_{m, N}(k-1)+e_{m, N}^{p}(k) e_{m, N+1}^{H}(k)$ & 1 \\
\hline$\alpha_{m, N}(k)=\alpha_{m, N+1}(k)-a_{m, N}^{s}(k) a_{m, N+1}^{H}(k)$ & 1 \\
\hline$\alpha_{m+1, N}(k)=\alpha_{m, N}(k)-\rho_{m, N}(k) \beta_{m, N}^{-1}(k-1) \rho_{m, N}^{H}(k)$ & 1 (1 divide) \\
\hline$\gamma_{m+1, N+1}(k)=\gamma_{m, N+1}(k-1)-e_{m, N+1}^{H}(k) \alpha_{m, N+1}^{-1}(k) e_{m, N+1}(k)$ & 1 (1 divide) \\
\hline$\tilde{C}_{m+1, N+1, k}=\left[\begin{array}{ll}0 & \tilde{C}_{m, N+1, k-1}\end{array}\right]-\frac{e_{m, N}^{\mu n}(k)}{\alpha_{m, N}(k)} A_{m, N, k-1}$ & $m$ \\
\hline$A_{m, N, k}=A_{m, N+1, k}-a_{m, N}^{s}(k)\left[\begin{array}{ll}0 & D_{m, N+1, k-1}\end{array}\right]$ & $m$ \\
\hline$A_{m+1, N, k}=\left[\begin{array}{ll}A_{m, N, k} & 0\end{array}\right]-\frac{\rho_{m, N}(k)}{\beta_{m, N}(k-1)}\left[\begin{array}{ll}0 & B_{m, N, k-1}\end{array}\right]$ & $m$ \\
\hline$B_{m+1, N, k}=\left[\begin{array}{ll}0 & B_{m+1, N, k-1}\end{array}\right]-\frac{\rho_{m, N}^{H}(k)}{\alpha_{m, N}(k)}\left[\begin{array}{lll}A_{m, N, k} & 0\end{array}\right]$ & $m$ \\
\hline$D_{m+1, N+1, k}=\left[\begin{array}{ll}0 & D_{m, N+1, k-1}\end{array}\right]-\frac{a_{m, N+1}^{H}(k)}{\alpha_{m, N+1}(k)} A_{m, N+1, k}$ & $m$ \\
\hline$\beta_{m+1, N}(k)=\beta_{m, N}(k-1)-\rho_{m, N}^{H}(k) \alpha_{m, N}^{-1}(k) \rho_{m, N}(k)$ & 1 \\
\hline$\delta_{m+1, N+1}(k)=\delta_{m, N+1}(k-1)-a_{m, N+1}^{H}(k) \alpha_{m, N+1}^{-1}(k) a_{m, N+1}(k)$ & 1 \\
\hline$\left[\begin{array}{c}E_{N, m, N+1, k}^{H} \\
V_{N, m+1, N+1, k}^{H}\end{array}\right]=\mathbf{T}_{1, k} \mid \begin{array}{c}e_{m, N}^{p}(k) \quad E_{N-1, m, N, k-1}^{H} \\
V_{N, m, N+1, k-1}^{H}\end{array}$ & $2 N$ \\
\hline$\left[\begin{array}{c}E_{N, m, N, k}^{H} \\
Y_{N, m+1, N+1, k}^{H}\end{array}\right]=\mathbf{T}_{3, k}\left[\begin{array}{c}E_{N, m, N+1, k}^{H} \\
Y_{N, m, N+1, k-1}^{H}\end{array}\right]$ & $2 N$ \\
\hline$\left[\begin{array}{l}E_{N, m+1, N, k}^{H} \\
R_{N, m+1, N, k}^{H}\end{array}\right]=\mathbf{T}_{5, k}\left[\begin{array}{c}E_{N, m, N, k}^{H} \\
R_{N, m, N, k-1}^{H}\end{array}\right]$ & $2 N$ \\
\hline Total (Prediction) & $6 N+11 m+7$ (3 divides) \\
\hline \multicolumn{2}{|l|}{ Joint Process Extension } \\
\hline$\epsilon_{m, N}^{p}(k)=d(k)-W_{N, m, N, k-1} X_{N, k}$ & $N$ \\
\hline $\mathcal{E}_{m+1, m, N, k}^{H}=\left[\begin{array}{ll}\epsilon_{m, k}^{p}(k) & (1-\mu) \mathcal{E}_{m, m, N, k-1}^{H}\end{array}\right.$ & $m$ \\
\hline$\zeta_{m, N}(k)=\mathcal{E}_{m+1, m, N, k}^{H} A_{m, N, k}^{H}$ & $m$ \\
\hline$G_{N, m+1, N, k}=(1-\mu) G_{N, m, N, k-1}+\mu \frac{\zeta_{m, N}(k)}{\alpha_{m, N}(k)} E_{N, m, N, k}^{H}$ & $2 N+1$ (1 divide) \\
\hline$W_{N, m+1, N, k}=W_{N, m, N, k-1}+G_{N, m+1, N, k}$ & - \\
\hline \multicolumn{2}{|l|}{ Note: $\quad m=k-k_{s}$} \\
\hline Total (Prediction and Joint Process Estimation) & $9 N+13 m+8$ (4 divides) \\
\hline
\end{tabular}

where the definitions of $\eta$ and $\zeta$ follow from (24). The exact update of the $\Upsilon$ vector can be achieved when the sliding window FTF algorithm of order $m$ is used to compute the predictors $A$ and $B$. An approximate version can be found when the exponentially windowed FTF algorithm is employed as discussed in the next section. The complete algorithm can be found in Table IV with reintroduced indices on $S$, where they have the same meaning as on $W$. The complexity is comparable to the NLMS algorithm, where the dominant figure is $2 N$.
Unfortunately, a growing window version of the EURLS is not tractable because the entries of the $\Upsilon$ vector are needed at various previous time instants, which is not practical either to compute or to store.

\section{APPROXIMATIONS AND SPECIAL CASES OF THE URLS ALGORITHM}

Many suboptimal adaptive filtering algorithms in the sense of underdetermined least-squares may be found, depending on the degrees of approximations imposed on various quantities 
TABLE IV

EURLS-FTF-SWC ALGORITHM

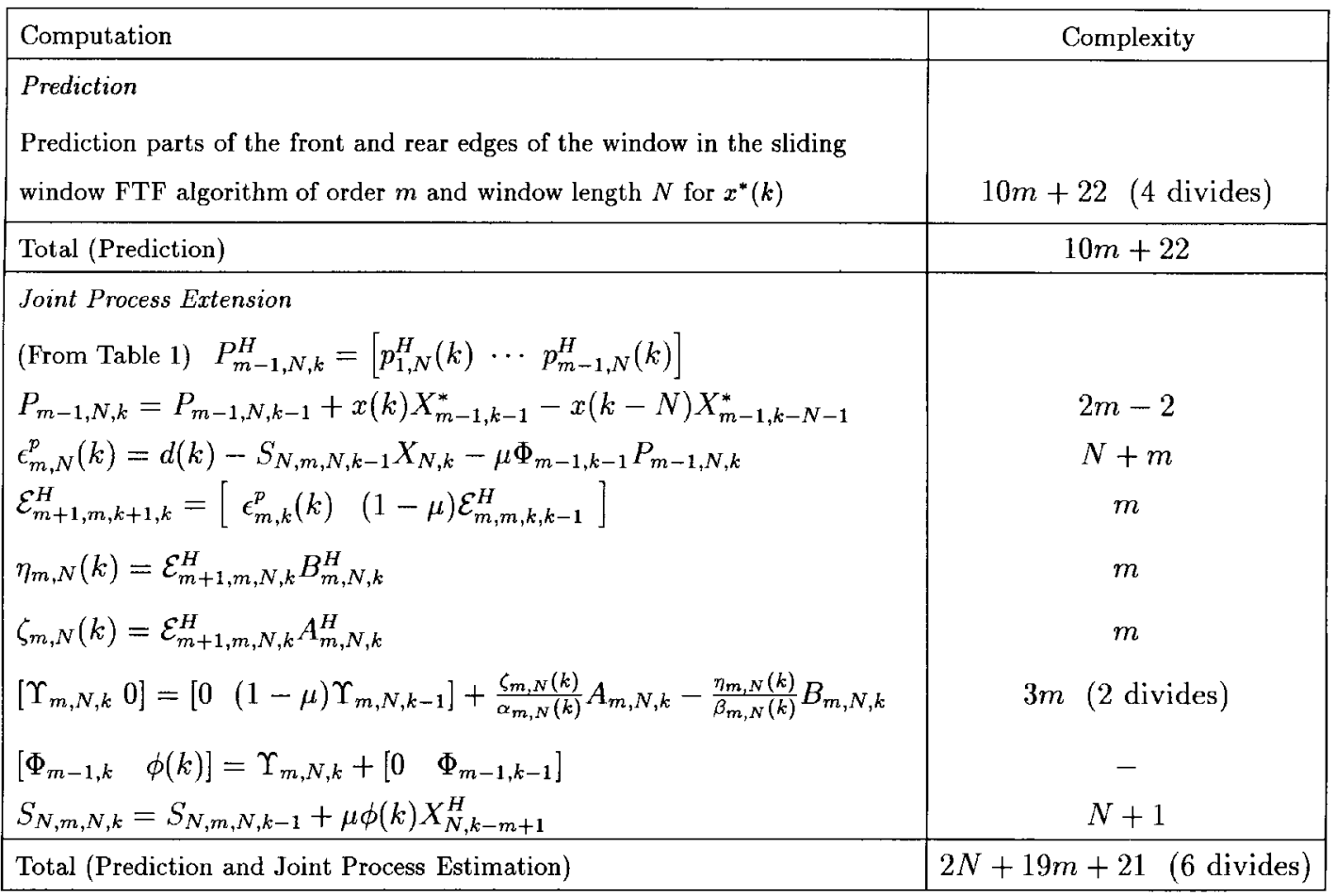

of the optimal URLS algorithm. Below, we describe some alternatives and show that some adaptive algorithms that have appeared in the literature are essentially suboptimal approximations of the URLS algorithm.

\section{A. Approximation in Prediction Error Vectors}

Efficient approximations of the URLS algorithms can be found by approximating the prediction error vectors. For example, consider the forward prediction error vector

$$
E_{N, m,-, k}^{H}=A_{m,-, k} \mathbf{X}_{m+1, N, k}^{H} .
$$

The time update of this prediction error vector requires $O(N)$ operations. It may be approximated as

$$
\left.\begin{array}{rl}
\hat{E}_{N, m,-, k}^{H} \triangleq & {\left[\begin{array}{lll}
e_{m,-}(k) & e_{m,-}(k-1) & \cdots
\end{array}\right.} \\
& e_{m,-}(k-N+2) \quad e_{m,-}(k-N+1)
\end{array}\right]
$$

where the elements of $\hat{E}$ have already been computed at previous instants. Thus, the update of the $G$ vector in (25) becomes

$$
\begin{aligned}
G_{N, m,-, k}= & (1-\mu) G_{N, m,-, k-1} \\
& +\mu \frac{1}{\alpha_{m,-}(k)} \zeta_{m,-}(k) \hat{E}_{N, m,-, k}^{H} \\
& -\mu \frac{1}{\beta_{m,-}(k)} \eta_{m,-}(k) \hat{R}_{N, m,-, k}^{H}
\end{aligned}
$$

Hence, $O(N)$ operations to update $E$ and $R$ may be dropped. This approximation is applicable to all prediction error vectors. As a result of this approximation, for example, the dominant figure in the complexity of the URLS-FTF-SWC algorithm is reduced to $4 N$ from $14 N$. It can be seen that in the URLSFTF algorithm, the update of prediction error vectors consumes most of the computational effort. Since the prediction error vectors are replaced with a time-series signal of the type defined in (81), the URLS algorithm reduces to an instrumental variable technique. The instrumental variables in the approximate URLS algorithm can be identified as the time-series signals of the a posteriori forward and backward prediction residuals.

In the following paragraphs, some further approximations are discussed. When the computation of the transversal filter $A$ is further approximated by using the LMS algorithm and the backward prediction error filter $B$ is discarded, a filtered-X LMS algorithm is obtained [26], [27]. In addition, the $m$ element prediction error vector $\mathcal{E}$ is approximated by $m-1$ a priori error samples of previous time instants except for the first element. Let $\hat{A}$ denote the forward predictor without the leading 1 . Hence, the update equations are

$$
\begin{aligned}
\hat{A}_{m,-, k} & =\hat{A}_{m,-, k-1}-\mu_{1} e_{m,-}^{p}(k) X_{m, k-1}^{T} \\
W_{N, m,-, k} & =W_{N, m,-, k-1}+\mu_{2} \hat{\zeta}_{m,-}(k) \hat{E}_{N, m,-, k}^{H}
\end{aligned}
$$

where

$$
\begin{aligned}
e_{m,-}^{p}(k) & =x^{*}(k)+\hat{A}_{m,-, k-1} X_{m, k-1}^{*} \\
\epsilon_{m,-}^{p}(k) & =d(k)-W_{N, m,-, k-1} X_{N, k} \\
\hat{\mathcal{E}}_{m, m,-, k}^{H} & \triangleq\left[\epsilon_{m,-}^{p}(k) \cdots \epsilon_{m,-}^{p}(k-m+1)\right] \\
\hat{\zeta}_{m,-}(k) & =\hat{\mathcal{E}}_{m, m,-, k}^{H} \hat{A}_{m,-, k}^{H} .
\end{aligned}
$$

The averaged LMS (ALMS) algorithm [28] is the crudest approximation to the URLS algorithm as it assumes $\mathbf{U}_{m, N, k}^{-1}=$ $\mathbf{I}_{m}$ and then proceeds to average $m$ consecutive $\epsilon_{-,-}^{p}(i) X_{N, i}$, $i=k, \ldots, k-m+1$ products. The assumption about the 
TABLE V

APPROXIMATE URLS-FTF-SWC ALgORITHM

\begin{tabular}{|c|c|}
\hline Computation & Complexity \\
\hline \multicolumn{2}{|l|}{ Prediction } \\
\hline Prediction part of the exponential window FTF algorithm of order $m$ for $x^{*}(k)$ & $5 m+13$ (2 divides) \\
\hline$\left[\begin{array}{c}E_{N, m, k+1, k}^{H} \\
V_{N, m+1, k+1, k}^{H}\end{array}\right]=\mathbf{T}_{\mathbf{1}, k}\left[\begin{array}{c}e_{m, k}^{p}(k) \quad E_{N-\mathbf{1}, m, k, k-\mathbf{1}}^{H} \\
V_{N, m, k, k-1}^{H}\end{array}\right.$ & $2 N$ \\
\hline 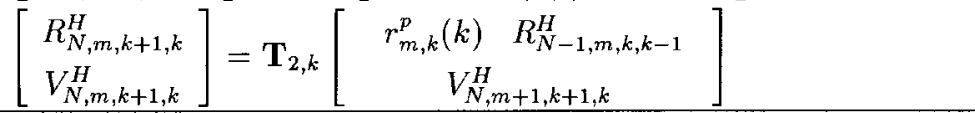 & $3 N$ \\
\hline Total (Prediction) & $5 N+5 m+13$ (2 divides) \\
\hline \multicolumn{2}{|l|}{ Joint Process Extension } \\
\hline$\epsilon_{m, k}^{p}(k)=d(k)-W_{N, m, k, k-1} X_{N, k}$ & $N$ \\
\hline $\mathcal{E}_{m+1, m, k+1, k}^{I}=\left[\begin{array}{ll}\epsilon_{m, k}^{p}(k) & (1-\mu) \mathcal{E}_{m, m, k, k-1}^{H}\end{array}\right.$ & $m$ \\
\hline$\eta_{m, k+1}(k)=\mathcal{E}_{m+1, m, k+1, k}^{H} B_{m, k+1, k}^{H}$ & $m$ \\
\hline$\zeta_{m, k+1}(k)=\mathcal{E}_{m+1, m, k+1, k}^{H} A_{m, k+1, k}^{H}$ & $m$ \\
\hline \multicolumn{2}{|l|}{$G_{N, m, k+1, k}=(1-\mu) G_{N, m, k, k-1}+\mu \frac{\zeta_{m, k+1}(k)}{\alpha_{m, k+1}(k)} E_{N, m, k+1, k}^{H}$} \\
\hline$-\mu \frac{\eta_{m, k+1}(k)}{\beta_{m, k+1}(k)} R_{N, m, k+1, k}^{H}$ & $3 N+2$ (2 divides) \\
\hline$W_{N, m, k+1, k}=W_{N, m, k, k-1}+G_{N, m, k+1, k}$ & - \\
\hline Total (Prediction and Joint Process Estimation) & $9 N+8 m+15$ (4 divides) \\
\hline
\end{tabular}

prediction error vector $\mathcal{E}$ in the filtered-X LMS algorithm is also valid in the ALMS. Thus, we have

$$
W_{N,-,-, k}=W_{N,-,-, k-1}+\frac{\mu}{m} \hat{\mathcal{E}}_{m,-,-, k}^{H} \mathbf{X}_{m, N, k}^{H} .
$$

It turns out that the so-called conjugate gradient $(\mathrm{CG})$ approach in adaptive filtering [10] is equivalent to solving the URLS problem [5]. When the optimization problem in Section II-B is solved at each instant by recursively generating conjugate vectors in the affine subspace of $m$ input vectors, the solution $W_{N,-,-, k}$ is the same as that of (3). Note that there is no recursive propagation of any vectors or matrices and no step-size in the CG approach. The fast CG algorithm [10] was introduced to save computation time in the computationally complex CG approach and is an approximation to the URLS algorithm such that the conjugacy of the recursively generated vectors are lost; hence, the resulting affine subspace is approximately equal to that spanned by input vectors.

\section{B. Approximation in Transformations}

It is also possible to make some simplifications in the updates of the prediction error vectors that yield a reduced number of transformations to compute the prediction error vectors and filters. An obvious approach is to approximate the sliding window FTF algorithm, from which the prediction residuals are obtained for the update process, with the exponentially windowed FTF to reduce the computational load by half. Such an approximation corresponds to the use of the matrix inversion lemma with exponential windowing to estimate the covariance matrix in (7), which also help improve conditioning of the covariance matrix [6]. This can be achieved by discarding the update of the trailing edge of the window and adding an exponential forgetting factor. Thus, the prediction is performed over an exponentially weighted window that is practically equivalent to a finite length window for stationary input signals [29]. This reduces the computational load, yet it preserves in a sense the sliding effect of the window.

The derivation yields

$$
\begin{gathered}
{\left[\begin{array}{c}
A_{m, k+1, k} \\
\tilde{C}_{m+1, k+1, k}
\end{array}\right]=\left[\begin{array}{cc}
1 & e_{m, k+1}(k) \\
\frac{\lambda^{-1} e_{m, k}^{p}(k)}{\alpha_{m, k}(k-1)} & 1
\end{array}\right]\left[\begin{array}{c}
A_{m, k, k-1} \\
{\left[\begin{array}{c}
0 \\
\tilde{C}_{m, k, k-1}
\end{array}\right]}
\end{array}\right]} \\
{\left[\begin{array}{c}
B_{m, k+1, k} \\
{\left[\tilde{C}_{m, k+1, k}\right.}
\end{array}\right]=\mathbf{T}_{2, k}\left[\begin{array}{c}
B_{m, k, k-1} \\
\tilde{C}_{m+1, k+1, k}
\end{array}\right]}
\end{gathered}
$$

where $\mathbf{T}_{1, k}$ in (86) is modified slightly by adding the forgetting factor. In addition, note the change of the window index from $N$ to $k+1$ on various quantities. This reflects the increase in the window length at each iteration as a result of the use of the exponentially windowed FTF (FTF-EW) algorithm. The updates of the prediction errors become

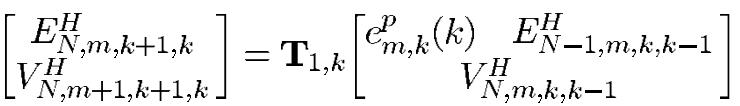

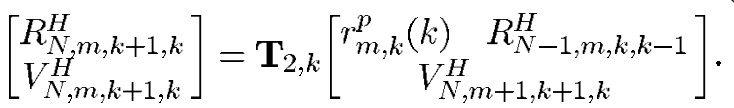

The derivation shows that the prediction error vectors can be updated with a smaller number of operations, namely, with two transformations instead of four transformations when compared with the exact FTF-SWC algorithm, thereby leading 
to a reduced number of operations. The complete algorithm is summarized in Table V.

An approximate version of the URLS-FTF-GWC algorithm can also be derived in which the update of the rear edge of the window is discarded. If the prediction error filter $D$ is not included in the recursions, the estimates of all quantities in the URLS-FTF-GWC algorithm will not be calculated across a window of length $N$, which will then be replaced by an exponentially weighted window. Hence, the transformation $\mathbf{T}_{3, k}$ will not be included in Table III.

\section{Special Case}

In the special case $\mu=1$, the $G$ vector simplifies to

$$
G_{N, m, N, k}=\frac{1}{\alpha_{m-1, N}(k)} E_{N, m-1, N, k}^{H}
$$

in which it is seen that the dependence on $G_{N, m, N, k-1}$ and on the backward prediction variables is dropped. However, the computation of $E_{N, m-1, N, k}^{H}$ requires other prediction error vectors $R, V$, and $Y$ at the intermediate stages. The sole difference is that the prediction is carried out for prediction order $m-1$ instead of $m$. Furthermore, it is observed that the prediction error filters $A, B, C$, and $D$ are not required in the update.

\section{Practical IsSUES}

In this section, we discuss problems that may arise in practical implementations of the URLS algorithm. In its direct implementation (6) with the matrix inversion lemma, a potential source of numerical problem is the evaluation of the inverse of the covariance matrix, which is also a problem of the standard RLS algorithm. If this matrix is badly conditioned, as in many practical cases, the widely used Tikhonov regularization technique provides a solution. Before the first application of the matrix inversion lemma, the inverse of the covariance matrix is initialized to a diagonal matrix of the form $\mathbf{U}_{m, N,-1}=c \mathbf{I}_{m}$, where $c$ is a small constant so that the eigenvalues of the covariance matrix are ensured to reside above $c$. This works even for signals that are not persistently exciting. In the sliding window update of the covariance matrix, the effect of $c$ never dies out, contrary to the exponentially windowed update in which weighting causes the regularization to diminish in time. Hence, in this respect, the URLS algorithm is safe to use as shown in the simulations. Note that when the URLS algorithm is regularized, the relationships presented in Section II-B are only approximately true. If $m=1$ (NLMS algorithm), the parameter $c$ can be identified as the safety parameter introduced for ill-conditioned cases in which the power of the input signal falls close to zero and jeopardizes the stability of the algorithm by leading to large step sizes. The exponential window approximation discussed earlier, which is closely related to the one in [6], eases the erratic behavior of the basic algorithm, although the responsiveness to time-varying systems is to some extent sacrificed. This is not a solution in itself; if the input is barely persistently exciting, bad conditioning still occurs.
The effect of the aforementioned regularization technique is reflected within the URLS-FTF-SWC algorithm in an implicit way by initializing the residual powers as $\alpha_{m, N}(-1)=$ $\beta_{m, N}(-1)=c$. Thus, the influence of the parameter $c$ does not decay and enhances the conditioning of the inverse covariance matrix [19]. The initialization of the prediction parts of the URLS-FTF algorithms are identical to those of the FTF algorithms [19], [20]. Stabilization is a more involved issue. However, one can use the stabilization procedures of Slock reported in [30] and increase the computational complexity by a constant factor in the prediction order part of the URLSFTF algorithm, i.e., the complexity of the prediction part of the URLS-FTF-SWC algorithm becomes $10 N+12 m+24$ (four divides). Another solution for the numerical stability is the straightforward approach of running two sliding window covariance estimates and intermittent initialization and restart. The source of problem in the URLS-FTF-SWC algorithm, namely, the joint update of the backward prediction filter and Kalman gain, is not included in the URLS-FTF-GWC algorithm; therefore, we may expect better numerical performance. The above regularization technique would also be valid in this case. The numerical behavior of the URLS-FTF algorithms are governed mainly by the prediction parts. The joint process extension parts have LMS-like updates of the $G$ vector, and LMS-type recursions are known to be numerically stable, except for the subtle problem of parameter drift [31], which may happen for certain input excitations. However, the other problem peculiar to LMS (stalling phenomenon) may occur if $\mu$ is chosen too small.

\section{SimULATION EXAMPLES}

Various experiments have been performed to assess the performance of the URLS algorithms along with the stabilized sliding window FTF (FTF-SW) and exponentially weighted FTF (FTF-EW) algorithms. The stabilization procedure of [30] has been used. The NLMS algorithm has been implemented as the first-order URLS algorithm. For the higher order URLS algorithms, the URLS-FTF formulation has been used. The fast Newton transversal filters (FNTF) algorithm [32] has also been included in the simulations as it is similar to the URLS algorithm in the sense that whitening filters are used on the input. In Section III, we have explained the fact that the effective prediction order of the input is one less than the order of the URLS algorithm. The order of the FNTF algorithm equals the effective prediction size of the input so that the zeroth-order FNTF algorithm corresponds to the NLMS algorithm. We have also indicated the effective prediction order of the input in the tables and figures. It would be possible to use acceleration techniques on algorithms such as reported in [33] for the FNTF algorithm and in [34], which may be adapted to the URLS algorithm to have a variable step size. We have avoided such techniques to compare the basic algorithms. All simulations were carried out with the full floating-point precision of a Sparc20 workstation.

\section{A. Experiments with Synthetic Signals}

Three experiments are reported in this part that are presented under three sections, namely, tracking a time-varying reference 


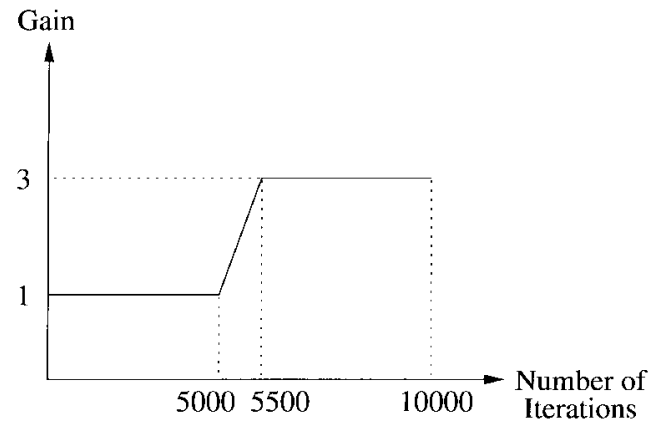

Fig. 3. Gain variation for the tracking experiment.

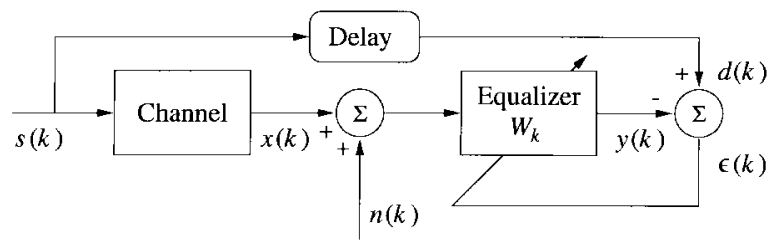

Fig. 4. Baseband channel equalization model.

model in system identification, tracking a time-varying communications channel in equalization, and response to a spectral change in the input.

1) Tracking in System Identification: In this part, we have identified a time-varying system with the setup of Fig. 2. The adaptive filter and reference system have $N=64$. A stationary 10th-order autoregressive input signal is chosen. The poles of the input signal are located at $0.65 \mp 0.7 j,-0.8 \mp 0.4 j$, $0.2 \mp 0.9 j,-0.5 \mp 0.8 j$, and $0.8 \mp 0.5 j$ on the $z$ plane. A piecewise linear gain of the form in Fig. 3 is introduced at the output of the reference system to simulate the time-varying system. The average SNR level is set to $20 \mathrm{~dB}$. The results obtained after 20 realizations of the experiment are averaged. The system mismatch

$$
s(k)=\frac{\left\|W_{\mathrm{opt}}-W_{N, m, N, k}\right\|^{2}}{\left\|W_{\mathrm{opt}}\right\|^{2}}
$$

where $W_{\text {opt }}$ denotes the optimal system, is computed as a function of time and plotted in the figures. All algorithms are initialized to the average power of the input signal $\sigma^{2}$, except for the FTF-SW algorithm, in which the initial condition is chosen as $0.01 \sigma^{2}$, and the forgetting factors for the FNTF and FTF-EW algorithms are set to $\lambda=1-1 / 3 N$. The window length of the FTF-SW algorithm is $3 N$.

Figs. 5 to 7 show the evolution of the system mismatches for all algorithms. We can observe that tracking capability of the URLS-FTF algorithm is acceptable. The best tracking algorithm in this case is the FTF-SW algorithm. We then have the URLS-FTF algorithm of the eighth effective prediction order of the input with $\mu=0.08$, which has approximately the same level of misadjustment as the FTF-EW algorithm. Simulations in various conditions indicate that the performance of the FNTF and URLS algorithms can be brought close for AR driving signals by tuning the forgetting factor in the FNTF and the step size in the URLS algorithms. Another observation is that the URLS algorithm is sensitive to additive noise that is compensated to some extent by choosing smaller step sizes.

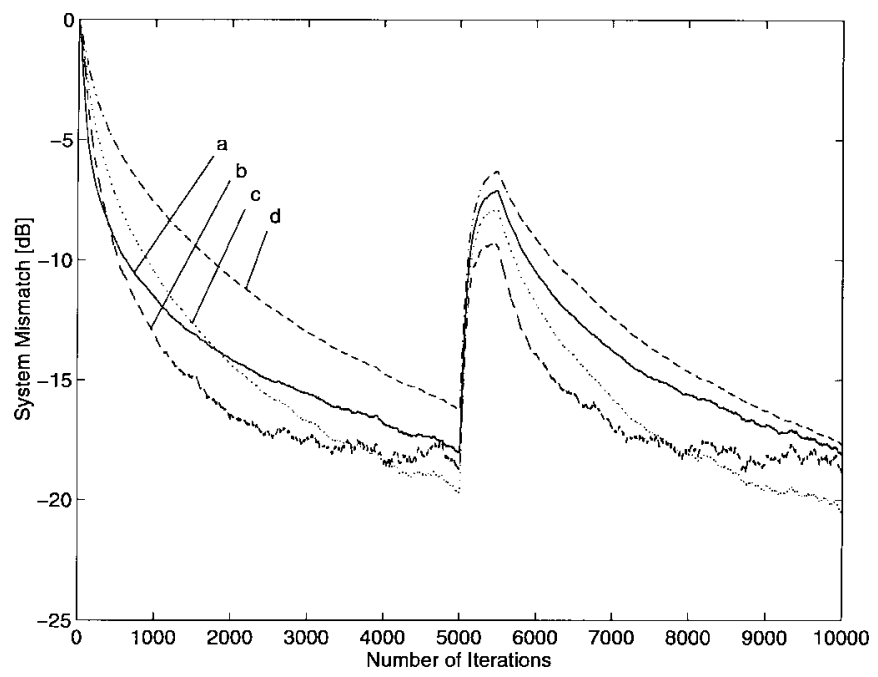

Fig. 5. Tracking in system identification: Effective prediction order of input is 2. (a) FNTF. (b) URLS-FTF $\mu=0.4$. (c) $\mu=0.2$. (d) $\mu=0.1$.

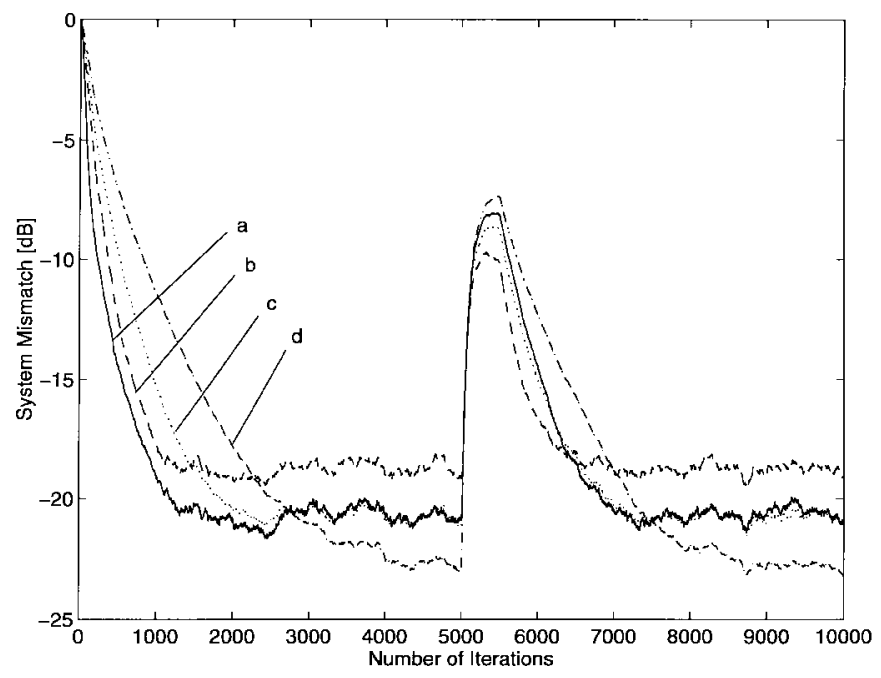

Fig. 6. Tracking in system identification: Effective prediction order of input is 8. (a) FNTF. (b) URLS-FTF $\mu=0.08$. (c) $\mu=0.05$. (d) $\mu=0.03$.

2) Tracking in Channel Equalization: The second experiment deals with the tracking of a time-varying communications channel. The setup in Fig. 4 is used. The channel is chosen to be nonminimum phase of the form $\left[\begin{array}{lllll}0.1 & 0.2 & -0.4 & 0.6 & -0.9\end{array}\right]$. The equalizer has $N=31$. The input is a binary pulse amplitude modulation system with iid distributed samples of $\mp 1$. The SNR level in the channel is set to $30 \mathrm{~dB}$. The third coefficient of the channel is sign reversed at sample number 1000 to simulate a time-varying channel. The results are obtained from the average of 20 realizations of the experiment. The algorithms are evaluated according to the interference to signal ratio (ISR) measurements. ISR is defined for $M$-level pulse amplitude modulation schemes as

$$
\operatorname{ISR}=(M-1) \sum_{i \neq v} \frac{\left|t_{i}\right|}{\left|t_{v}\right|}
$$

where $t_{i}$ are elements of the convolution of channel impulse response and equalizer, and $t_{v}=\max _{i}\left|t_{i}\right|$ [35]. When the 


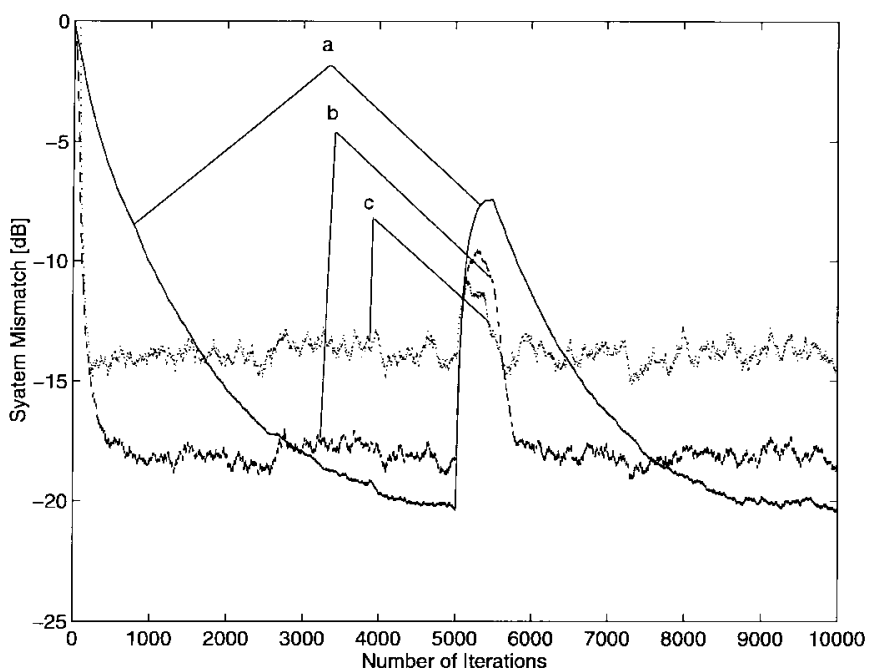

Fig. 7. Tracking in system identification. (a) NLMS $\mu=1$. (b) FTF-EW. (c) FTF-SW.

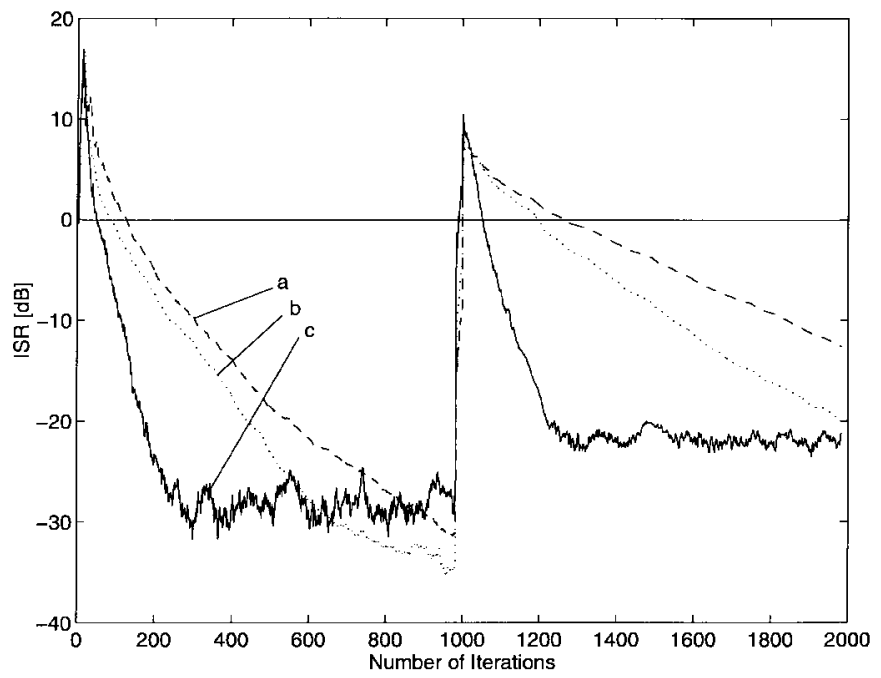

Fig. 8. Tracking in channel equalization. Effective prediction order of input is 2. (a) FNTF. (b) URLS-FTF $\mu=0.2$. (c) $\mu=1$.

residual intersymbol interference at the equalizer output is small enough to yield an open-eye equalizer output, ideal sequence recovery can be achieved by a quantizer. If the ISR is below $0 \mathrm{~dB}$, then the eye of the equalizer output is open. If the ISR is above $0 \mathrm{~dB}$, then the equalizer output has a closed eye, and therefore, decision errors will occur. The choice of parameters is the same as in the previous subsection.

Figs. 8 to 10 show the evolution of the ISR's for all algorithms. The important parameter in channel equalization simulations is the time spent above the $0 \mathrm{~dB}$ line. We can observe that the URLS-FTF algorithm outperforms the FNTF algorithm such that the recovery time of the URLS-FTF algorithm is much shorter than the FNTF algorithm after the channel characteristics have changed. The higher order URLSFTF algorithm, in particular, has the best performance. The unsatisfactory performance of the FNTF algorithm can be attributed to the fact that the inverse of the covariance matrix

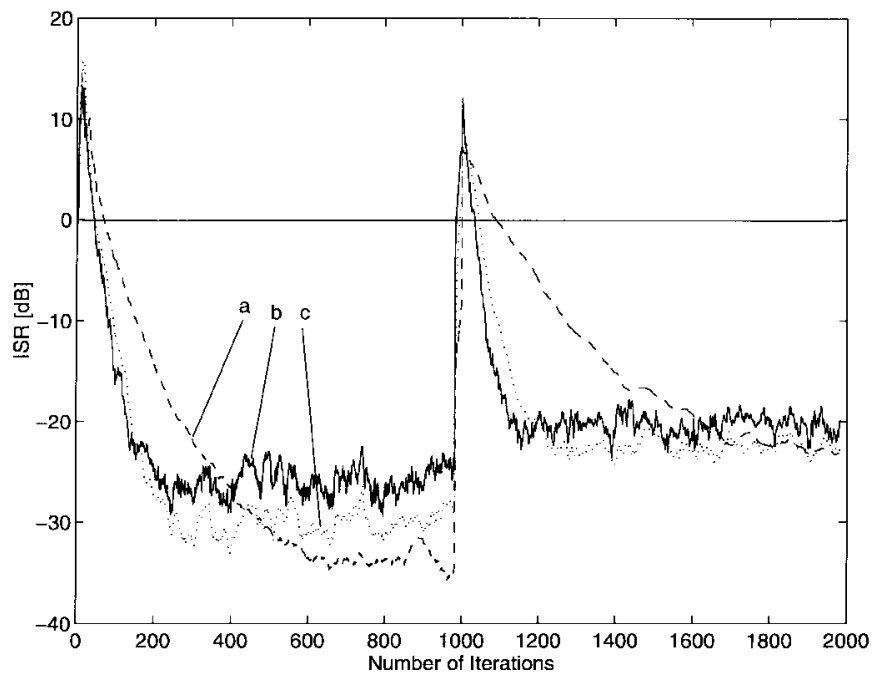

Fig. 9. Tracking in channel equalization. Effective prediction order of input is 8. (a) FNTF. (b) URLS-FTF $\mu=1$. (c) $\mu=0.2$.

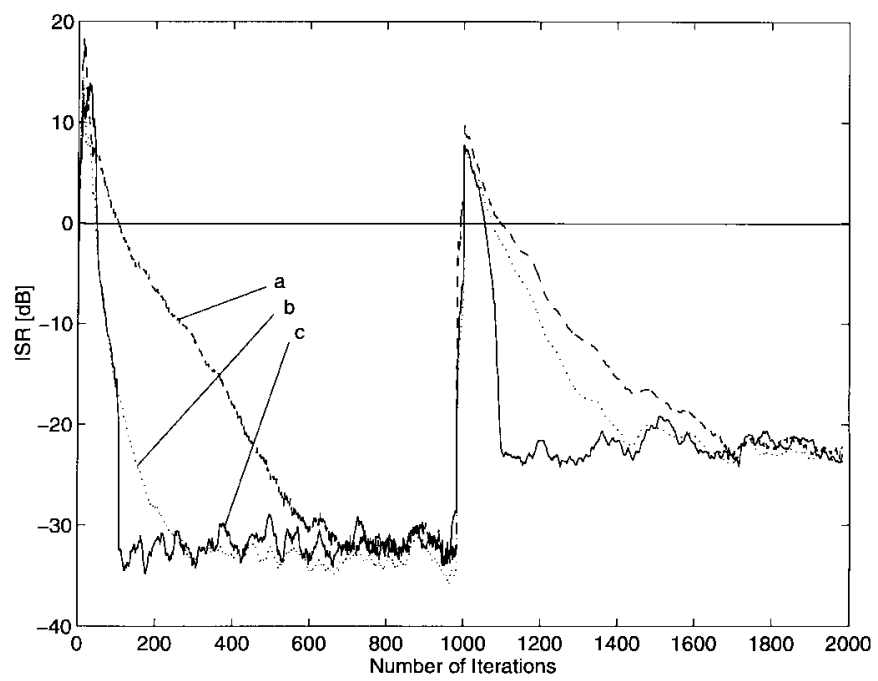

Fig. 10. Tracking in channel equalization. (a) NLMS $\mu=1$. (b) FTF-EW. (c) FTF-SW

is extrapolated by assuming an AR signal input, whereas the channel output is an MA signal.

3) Response to a Spectral Change in the Input: The setup and parameters of the first experiment is used, but the characteristics of the input signal is changed at sample 5000 such that it becomes a third-order AR signal having the same power with poles $0.7 \mp 0.6 j$ and 0.9 on the $z$ plane. The results are shown in Figs. 11 to 13. It can be seen that the URLS-FTF algorithm is less sensitive to such changes than the FNTF and FTF algorithms.

\section{B. Experiments with Real Recordings}

The algorithms are applied to the echo cancellation problem in hands-free telephone systems in car cockpits, which can be modeled as in Fig. 2, where the loudspeaker (far-end speech) and microphone signals are $x(k)$ and $d(k)+n(k)$, respectively. The echo path corresponds to the unknown system. 


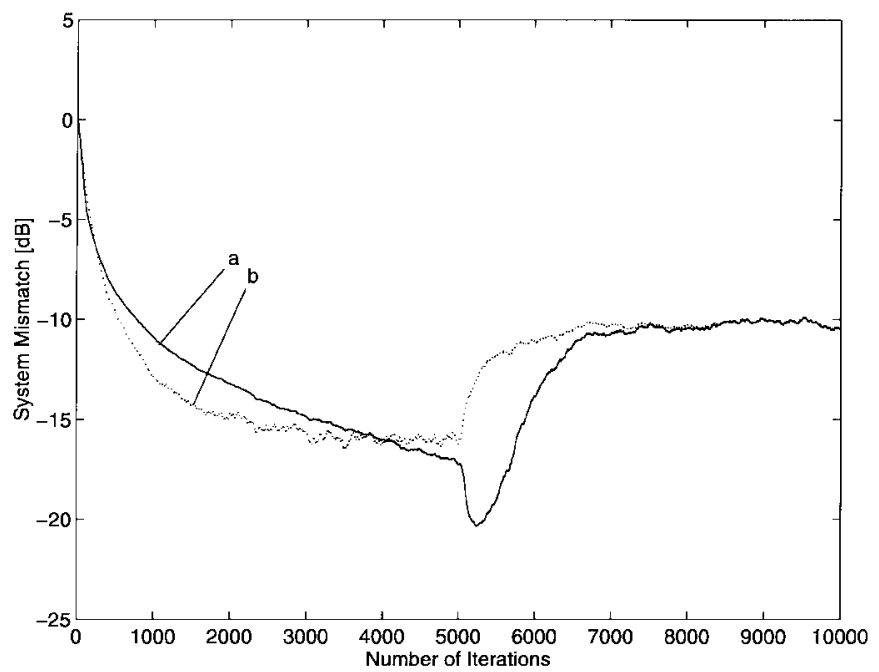

Fig. 11. Response to a spectral change: Effective prediction order of input is 2. (a) FNTF. (b) URLS-FTF $\mu=0.5$.

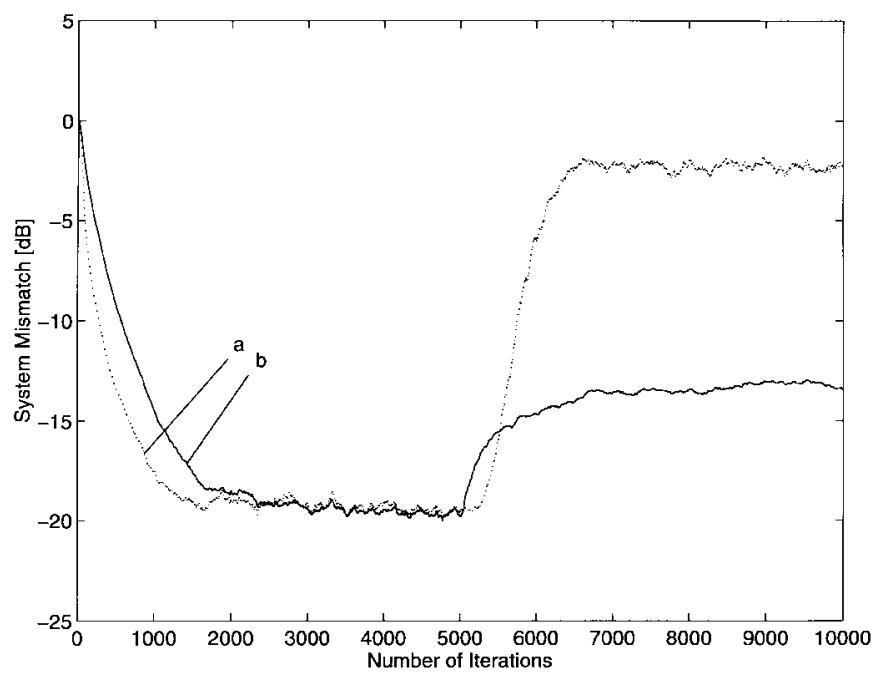

Fig. 12. Response to a spectral change. Effective prediction order of input is 8. (a) FNTF. (b) URLS-FTF $\mu=0.05$.

The loudspeaker and microphone signals were recorded in the cockpit of a Renault 25 car when the car was parked with the engine off and when the car was moving along a highway at $130 \mathrm{~km} / \mathrm{h}$ as a part of the echo database of the European Community research project FREETEL [36]. French speakers utter phonetically balanced sentences. Simulations were made on four recordings when the car was parked and on eight recordings when the car was moving, four of which are double talk situations, i.e., the near-end speaker is also active so that $n(k)$ equals the ambient noise added to the speech of the driver. Other recording conditions are as follows: loudspeaker type and position: Foster SE060 square loudspeaker at the driver's knee level; microphone type and position: Foster unidirectional, left upright relative to driver's position. The estimated echo path length has 128 samples. The algorithms are assessed according to echo return loss enhancement (ERLE) measurements. The ERLE is defined as

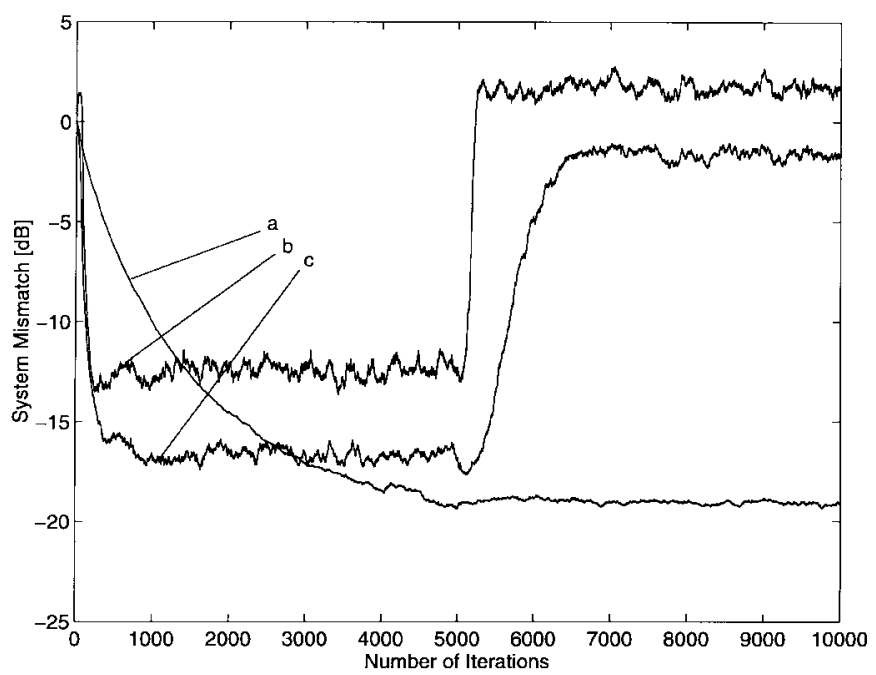

Fig. 13. Response to a spectral change. (a) NLMS $\mu=1$. (b) FTF-SW. (c) FTF-EW.

the ratio of the power of the microphone signal to the power of the error signal over segmented intervals, i.e.,

$$
\operatorname{ERLE}(n)=10 \log \left[\frac{\sum_{j=n L}^{(n+1) L-1}|d(j)|^{2}}{\sum_{j=n L}^{(n+1) L-1}|\epsilon(j)|^{2}}\right]
$$

where $n$ is the frame number. $L$ is chosen as 256 , which corresponds to $32 \mathrm{~ms}$ frames of $8 \mathrm{kHz}$ sampled signals. The mean of the ERLE across a recording is utilized to assess the performance of algorithms [37], which is similar to the FREETEL evaluation methodology [38]. The higher the ERLE, the better the algorithm performs to cancel echoes.

The choice of parameters for the algorithms are as follows: The forgetting factor of the FNTF algorithm is chosen as $\lambda=1-1 / 10 N$, and the initial input variance is set to $\sigma^{2} /(1-\lambda)$, where $\sigma^{2}$ is an a prior estimate of the input power as discussed in [33]. We have chosen $\sigma^{2}$ as the average power of the input speech. The forgetting factor of the FTFEW algorithm is the same as FNTF. The window length of the FTF-SW is $3 N$. Both FTF algorithms and the URLS-FTF algorithm are initialized to $\sigma^{2}$. The results are presented in Tables VI to VIII. In each table, the mean ERLE for four recordings are averaged.

We can observe that in all recordings, the best performance results from the URLS-FTF algorithm. In the noiseless situation, all algorithms produce similar results. The URLS-FTF algorithm is able to perform well in noisy conditions, whereas the other algorithms are badly affected. In the double talk situation, the speech of the near-end speaker can be regarded as noise and, ideally, must pass through the canceller untouched. This can be achieved by freezing the adaptive filter during double talk; otherwise, near-end speech can be distorted by the adaptive filter due to possible correlation between spectral characteristics of far and near-end speakers. In addtion, if the canceller is not frozen, near-end speech disturbs adaptive filter coefficients, yielding reduced ERLE. Yet in these simulations, 
TABLE VI

Mean erle Results Averaged Over Four Recordings When the Car Is Parked, Two Recordings for a Male Speaker, and Two Recordings for a Female Speaker

\begin{tabular}{cccccccccccc}
\hline & & \multicolumn{10}{c}{ Effective prediction order of the input } \\
\cline { 3 - 12 } & NLMS & 1 & 2 & 3 & 4 & 5 & 6 & 7 & 8 & 9 & 10 \\
\hline FNTF & & 11.92 & 12.09 & 12.09 & 12.12 & 12.07 & 12.06 & 12.08 & 12.11 & 12.13 & 12.12 \\
URLS-FTF $(\mu=1)$ & 10.83 & 12.95 & 13.34 & 13.28 & 13.48 & 13.36 & 13.41 & 13.34 & 13.36 & 13.36 & 13.28 \\
URLS-FTF $(\mu=0.9)$ & 11.07 & 13.38 & 13.73 & 13.66 & 13.80 & 13.65 & 13.66 & 13.58 & 13.59 & 13.58 & 13.49 \\
URLS-FTF $(\mu=0.8)$ & 11.25 & 13.72 & 14.02 & 13.94 & 14.02 & 13.87 & 13.84 & 13.74 & 13.73 & 13.71 & 13.63 \\
\hline FTF-EW & \multicolumn{11}{c}{13.50} \\
\hline FTF-SW & \multicolumn{11}{c}{10.17} \\
\hline
\end{tabular}

TABLE VII

Mean ERle Results Averaged Over Four Recordings When the Car Is Moving Along a Highway at 130 km/h, Two Recordings for a Male Speaker, Two Recordings for a Female Speaker

\begin{tabular}{|c|c|c|c|c|c|c|c|c|c|c|c|}
\hline & \multirow[b]{2}{*}{ NLMS } & \multicolumn{10}{|c|}{ Effective prediction order of the irput } \\
\hline & & 1 & 2 & 3 & 4 & 5 & 6 & 7 & 8 & 9 & 10 \\
\hline FNTF & & 1.00 & 0.87 & 0.86 & 0.89 & 0.91 & 0.93 & 0.95 & 0.95 & 0.94 & 0.94 \\
\hline URLS-F'TF $(\mu=1)$ & 3.50 & 9.51 & 9.33 & 9.00 & 8.64 & 8.30 & 7.98 & 7.76 & 7.58 & 7.35 & 7.12 \\
\hline URLS-F'F $(\mu=0.9)$ & 3.45 & 9.07 & 8.86 & 8.54 & 8.20 & 7.88 & 7.57 & 7.35 & 7.18 & 6.96 & 6.73 \\
\hline URLS-FTF $(\mu=0.8)$ & 3.35 & 8.55 & 8.34 & 8.03 & 7.71 & 7.12 & 7.11 & 6.90 & 6.73 & 6.52 & 6.30 \\
\hline F"l'F-EW & & & & & & 1.32 & & & & & \\
\hline FTF-SW & & & & & & 0.02 & & & & & \\
\hline
\end{tabular}

TABLE VIII

Mean ERle Results Averaged Over Four Recordings in a Double Talk Situation When the Car Is Moving Along a Highway at 130 km/h, Two Recordings for a Male Speaker, Two Recordings for a Female Speaker

\begin{tabular}{|c|c|c|c|c|c|c|c|c|c|c|c|}
\hline & \multirow[b]{2}{*}{ NLMS } & \multicolumn{10}{|c|}{ Effective prediction order of the input } \\
\hline & & 1 & 2 & 3 & 4 & 5 & 6 & 7 & 8 & 9 & 10 \\
\hline FNTF & & 0.93 & 0.74 & 0.73 & 0.72 & 0.72 & 0.73 & 0.73 & 0.74 & 0.73 & 0.72 \\
\hline URLS-FTF $(\mu=1)$ & 1.73 & 5.02 & 4.83 & 4.46 & 4.08 & 3.73 & 3.46 & 3.23 & 3.04 & 2.84 & 2.65 \\
\hline URLS-FTF $(\mu=0.9)$ & 1.80 & 4.85 & 4.61 & 4.25 & 3.88 & 3.53 & 3.26 & 3.04 & 2.84 & 2.65 & 2.45 \\
\hline URLS-F'TF $(\mu=0.8)$ & 1.82 & 4.63 & 4.35 & 4.00 & 3.65 & 3.31 & 3.04 & 2.82 & 2.63 & 2.44 & 2.24 \\
\hline FTF-EW & & & & & & 0.60 & & & & & \\
\hline FI'F-SW & & & & & & -0.12 & & & & & \\
\hline
\end{tabular}

the adaptive filter was operated continuously, which still led to satisfactory results for the URLS algorithm. When the error signal is listened to, the distortion on the near-end speech does not disturb the intelligibility. It may be surprising to see that the URLS algorithm, which is sensitive to observation noise, performs better in noisy recordings. We believe that the explanation lies in the fact that the tracking of the URLS algorithm is good and less affected by spectral changes, as shown in previous sections; in addition, lower order URLS algorithms are not very sensitive to observation noise. The reason why the step size is not chosen small in the NLMS and URLS-FTF algorithms, as one would expect, is due to the choice of initial condition (or regularization), the effect of which never dies out.

\section{CONCLUSION}

We have presented the concept and unified treatment of the URLS adaptive filtering, which is optimal in the least-squares sense for $\mu=1$ and lies between the NLMS and RLS adaptive filter algorithms. Hence, the URLS family algorithms also have intermediate behavior, yet they perform better in some cases. Fast transversal filter realizations that have the flexibility of dynamic prediction order change are provided; however, in order to exploit fully the potential of such algorithms, further research on the criteria, which can be used to detect the need for prediction order increment/decrement, is required. Some earlier published algorithms are shown to be suboptimal approximations of the exact URLS algorithm. A specific form of the URLS algorithm, called the EURLS algorithm, is also presented from an efficiency perspective in that it is not needed to compute the adaptive filter coefficients explicitly. Hence, the computational load is reduced, yet the exact solution of the URLS problem in terms of the prediction error is provided.

\section{REFERENCES}

[1] K. Ozeki and T. Umeda, "An adaptive filtering algorithm using an orthogonal projection to an affine subspace and its properties," Electron. Commun. Japan, vol. 67-A, no. 5, pp. 19-27, 1984. 
[2] H. Yasukawa, S. Shimada, and I. Furukawa, "Acoustic echo canceller with high speech quality," in Proc. ICASSP-87, 1987, pp. 2125-2128.

[3] P. Sommen and C. van Valburg, "Efficient realization of adaptive filter using an orthogonal projection method," in Proc. ICASSP-89, 1989, pp. 940-943.

[4] T. Furukawa, Y. Kimura, and H. Kubota, "A fast adaptive algorithm based on orthogonal projection onto multidimensional subspace," in Proc. ISSPA-90, 1990, pp. 27-31.

[5] D. Slock, "Underdetermined growing and sliding covariance fast transversal filter RLS algorithms," in Proc. EUSIPCO-92, 1992, pp. $1169-1172$.

[6] M. Montazeri and P. Duhamel, "A set of algorithms linking NLMS and RLS algorithms," in Proc. EUSPICO-94, 1994, vol. 2, pp. 744-747.

[7] M. Montazeri and P. Duhamel, "A set of algorithms linking NLMS and block RLS algorithms," IEEE Trans. Signal Processing, vol. 43, pp. 444-453, 1995.

[8] S. Gay and S. Tavathia, "The fast affine projection algorithm," in Proc. ICASSP-95, 1995, vol. 5, pp. 3023-3026.

[9] M. Tanaka, Y. Kaneda, S. Makino, and J. Kojima, "Fast projection algorithm and its step-size control," in Proc. ICASSP-95, 1995, pp 945-948.

[10] G. Boray and M. Srinath, "Conjugate gradient techniques for adaptive filtering," IEEE Trans. Circuits Syst.-I: Fundamental Theory Applications, vol. 39, no. 1, pp. 1-10, 1992.

[11] D. Marshall and W. Jenkins, "A fast quasi-Newton adaptive filtering algorithm," IEEE Trans. Signal Processing, vol. 40, no. 7, pp. 1652-1662, 1992.

[12] I. Karasalo, "Estimating the covariance-matrix by signal subspace averaging," IEEE Trans. Acoust., Speech, Signal Processing, vol. ASSP-34, no. 1 , pp. 8-12, 1986.

[13] P. Comon and G. Golub, "Tracking a few extreme singular-values and vectors in signal- processing," Proc. IEEE, vol. 78, no. 8, pp. $1327-1343,1990$

[14] G. Xu and T. Kailath, "Fast subspace decomposition," IEEE Trans. Signal Processing, vol. 42, no. 3, pp. 539-551, 1994.

[15] B. Yang, "Projection approximation subspace tracking," IEEE Trans. Signal Processing, vol. 43, no. 1, pp. 95-107, 1995.

[16] B. Baykal, "Underdetermined recursive-least squares adaptive filtering," Ph.D. dissertation, Imperial College, London, U.K., July 1995.

[17] F. Ling, "Givens rotation based least-squares lattice and related algorithms," IEEE Trans. Signal Processing, vol. 39, no. 7, pp. 1541-1551, 1991.

[18] J. Cioffi, "The block-processing FTF adaptive algorithm," IEEE Trans. Acoust., Speech, Signal Processing, vol. ASSP-34, no. 1, pp. 77-90, 1986.

[19] J. Cioffi and T. Kailath, "Windowed fast transversal filters adaptive algorithms with normalization," IEEE Trans. Acoust., Speech, Signal Processing, vol. ASSP-33, no. 3, pp. 607-624, 1985.

[20] _ , "Fast, recursive-least-squares transversal filters for adaptive filtering," IEEE Trans. Acoust., Speech, Signal Processing, vol. ASSP32, no. 2, pp. 304-337, 1984.

[21] B. Widrow and M. Lehr, "30 years of adaptive neural networks: Perceptron, madaline and backpropagation," Proc. IEEE, Special Issue on Neural Networks, vol. 78, 1990.

[22] G. Goodwin and K. Sin, Adaptive Filtering, Prediction and Control. Englewood Cliffs, NJ: Prentice-Hall, 1984.

[23] J. C. Magan, Ed., "Basic methods for echo cancellation, deliverable 3.123.1," Tech. Rep., Commission European Communities (FREETEL no. 6166), July 1993.

[24] R. Settineri and G. Favier, "Adaptive RLS lattice filters for fastly nonstationary signals," in Proc. ICASSP-90, 1990, pp. 1807-1810.

[25] B. Porat, B. Friedlander, and M. Morf, "Square root covariance ladder algorithms," IEEE Trans. Automat. Contr., vol. AC-27, pp. 813-829, 1982.

[26] M. Mboup, M. Bonnet, and N. Bershad, "LMS coupled adaptive prediction and system identification: A statistical model and transient mean analysis," IEEE Trans. Signal Processing, vol. 42, pp. 2607-2615, 1994.

[27] B. Widrow and S. Stearns, Adaptive Signal Processing. Englewood Cliffs, NJ: Prentice-Hall, 1985.

[28] J. Kim and L. D. Davisson, "Adaptive linear estimation for stationary $M$-dependent processes," IEEE Trans. Inform. Theory, vol. IT-21, pp. 23-31, 1975 .

[29] E. Eleftheriou and D. Falconer, "Tracking properties and steady-state performance of RLS adaptive filter algorithms," IEEE Trans. Acoust., Speech, Signal Processing, vol. ASSP-34, no. 5, pp. 1097-1109, 1986.

[30] D. Slock and T. Kailath, "Numerically stable fast transversal filters for recursive least squares adaptive filtering," IEEE Trans. Signal Processing, vol. 39, no. 1, pp. 92-113, 1991.
[31] W. Sethares, D. Lawrence, C. Johnson Jr., and R. Bitmead, "Parameter drift in LMS adaptive filters," IEEE Trans. Acoust., Speech, Signal Processing, vol. ASSP-34, no. 4, pp. 868-879, 1986.

[32] G. Moustakides and S. Theodoridis, "Fast Newton transversal filtersA new class of adaptive estimation algorithms," IEEE Trans. Signal Processing, vol. 39, no. 10, pp. 2184-2193, 1991.

[33] T. Petillon, A. Gilloire, and S. Theodoridis, "The fast Newton transversal filter: An efficient scheme for acoustic echo cancellation in mobile radio," IEEE Trans. Signal Processing, vol. 42, no. 3, pp. 509-518, 1994.

[34] V. Mathews and Z. Xie, "A stochastic gradient adaptive filter with gradient adaptive step size," IEEE Trans. Signal Processing, vol. 41, no. 6, pp. 2075-2087, 1993.

[35] S. Haykin, Ed., Blind Deconvolution. Englewood Cliffs, NJ: PrenticeHall, 1994

[36] J. Boudy, Ed., "Description of the databases: Background and additional materials, deliverable 1.3.1," Tech. Rep., Commission European Communities (FREETEL no. 6166), 1993.

[37] O. Tanrikulu, B. Baykal, and P. Naylor, Eds., "Advanced solutions for echo cancellation, deliverable 3.123.2," Tech. Rep., Commission European Communities (FREETEL no. 6166), 1994.

[38] J. C. Magan, Ed., "Methodology of evaluation and standards, deliverable 1.2," Tech. Rep., Commission European Communities (FREETEL no. 6166), July 1993

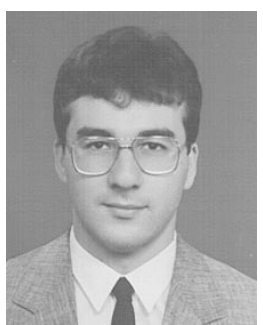

Buyurman Baykal (M'93) was born in Ankara, Turkey, in 1968. He received the B.Sc. degree (with High Hons.) from the Middle East Technical University, Turkey, in 1990 and the M.Sc. (with Distinction) and Ph.D. degrees from the Imperial College of Science, Technology, and Medicine of the University of London in 1992 and 1995, respectively, all in electrical and electronic engineering.

He held the British Council fellowship from 1991 to 1992 for postgraduate studies in the UK. He has been employed with Imperial College since 1993 as a research assistant and a research scientist, where he has been involved with various research projects on echo cancellation, noise reduction, and channel equalization. His research interests include blind and nonblind adaptive algorithms and their applications in communications, wireless systems, and hands-free communications.

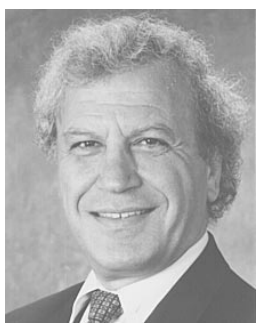

Anthony G. Constantinides (SM'78) is Professor of Signal Processing and the head of the Signal Processing and Digital Systems Section of the Department of Electrical and Electronic Engineering at Imperial College, London. He has been actively involved with research in various aspects of digital filter design, digital signal processing, and communications for a number of years. His research spans a wide range of digital signal processing, both from the theoretical as well as the practical points of view. His recent work has been directed toward the demanding signal processing problems arising from the area of telecommunications. He has published a range of books and papers in learned journals in the area of digital signal processing and its applications.

Dr. Constantinides served as the first president of the European Association for Signal Processing (EURASIP) and has contributed in this capacity to the establishment of the European Journal for Signal Processing. He has been on, and is currently serving as, a member of many technical program committees of IEEE international conferences. He organized the first ever international series of meetings on digital signal processing in London in 1967 and in Florence (with Vito Cappellini) since 1972. In 1985, he was awarded the Honor of Chevalier, Palmes Academiques, by the French Government, and in 1996, he was promoted to Officer, Palmes Academiques. He holds several visiting professorships and other fellowships and honors around the world. $\mathrm{He}$ presently is serving as a member of the Signal Processing Society Technical Committee on Neural Networks for Signal Processing of the IEEE Signal Processing Society. 